\title{
The morphology of minor axis gaseous outflows in edge-on Seyfert galaxies $\star, \star \star$
}

\author{
T. P. Robitaille ${ }^{1, \star \star \star}$, J. Rossa ${ }^{2,3}$, D. J. Bomans ${ }^{4}$, and R. P. van der Marel ${ }^{2}$ \\ 1 SUPA, School of Physics and Astronomy, University of St. Andrews, North Haugh, KY16 9SS St. Andrews, UK \\ e-mail: tr9@st-andrews.ac.uk \\ 2 Space Telescope Science Institute, 3700 San Martin Drive, Baltimore, MD 21218, USA \\ e-mail: marel@stsci.edu \\ 3 Department of Astronomy, University of Florida, 211 Bryant Space Science Center, PO Box 112055, Gainesville, FL 32611, USA \\ e-mail: jrossa@astro.ufl.edu \\ 4 Astronomisches Institut, Ruhr-Universität Bochum, Universitätsstrasse 150/NA7, 44780 Bochum, Germany \\ e-mail: bomans@astro.rub.de
}

Received 18 April 2006 / Accepted 20 December 2006

\section{ABSTRACT}

Context. Spiral galaxies often have extended outflows that permeate beyond the region of the disk. Such outflows have been seen both in starburst galaxies, actively star forming galaxies and galaxies with an AGN. In the latter galaxies it is unknown whether the large-scale outflows are driven by star formation activity or purely by the active nucleus.

Aims. The aim of our investigation is to study the frequency of extended minor-axis outflows in edge-on Seyfert galaxies to investigate the role of the AGN, the circumnuclear environment and star formation activity within the disk regions, and their importance for IGM enrichment on large scales.

Methods. We obtained optical narrowband imaging observations of a distance limited, northern hemisphere sample of 14 edge-on Seyfert spiral galaxies. Because of the distance-limited nature of the sample, it is restricted to relatively low-luminosity Seyfert galaxies. The data were obtained with BUSCA attached to the $2.2 \mathrm{~m}$ telescope at the Calar Alto observatory. Narrowband imaging in two different ionizational stages ( $\mathrm{H} \alpha$ and [O III]) was performed to attempt a discrimination between processes associated with the active nucleus and those connected to star forming activity within the disk. The median 3- $\sigma$ sensitivities for detection of high-latitude extended emission in the sample galaxies are $3.6 \times 10^{-17} \mathrm{erg} \mathrm{s}^{-1} \mathrm{~cm}^{-2} \operatorname{arcsec}^{-2}$ for the $\mathrm{H} \alpha$ images and $6.9 \times 10^{-17} \mathrm{erg} \mathrm{s}^{-1} \mathrm{~cm}^{-2} \mathrm{arcsec}^{-2}$ for the $[\mathrm{O}$ III] images. We use the data to study the distribution of extraplanar emission with respect to the AGN and the underlying disk H II regions.

Results. The H $\alpha$ morphology of the Seyfert galaxies is usually complex, but only in three out of 14 galaxies did we find evidence for minor axis disk outflows. At the sensitivity of our observations [O III] emission is generally detected only in the nuclear region. For Ark 79 we present the first evidence of a secondary nuclear component, best visible in the [O III] image, which has a linear separation from the primary nucleus of about $850 \mathrm{pc}$.

Conclusions. Overall, our results show that extraplanar emission of similar brightness and extent as in the previously known cases of NGC 3079 and NGC 4388 is not common in Seyfert galaxies of otherwise similar properties. Comparison with our previous results shows that for nearby edge-on spiral galaxies star formation may be a more powerful mechanism for producing DIG than AGN activity. While in general AGN activity undoubtedly plays some role in driving minor-axis outflows, this probably requires higher AGN luminosities than are encountered in our small distance-limited sample.

Key words. galaxies: nuclei - galaxies: ISM - galaxies: evolution - galaxies: Seyfert - galaxies: spiral - galaxies: structure

\section{Introduction}

The investigation of minor axis gaseous outflows in external galaxies by narrowband imaging is well established for starburst galaxies (e.g., Lehnert \& Heckman 1995, 1996a) and also for normal (non-starburst) galaxies (e.g., Rand 1996; Rossa \& Dettmar 2000; Miller \& Veilleux 2003; Rossa \& Dettmar $2003 b)$. In starburst galaxies the presence of gaseous outflows

* Based on observations collected at the Centro Astronómico Hispano Alemán (CAHA) at Calar Alto, operated jointly by the Max-Planck-Institut für Astronomie and the Instituto de Astrofísica de Andalucía (CSIC).

$\star \star$ Tables 2, 5 and Figs. 6-24 are only available in electronic form at http://www . aanda.org

$\star \star \star$ Also summer student and visitor at the Space Telescope Science Institute. is a common feature, where the gas and energy are being transported to the ambient intergalactic medium (IGM) from the nuclear region via starburst driven winds. In normal galaxies the presence of minor axis gaseous outflows is not as ubiquitous, but is however still a common phenomenon for galaxies exceeding a certain threshold of star formation activity in the underlying galaxy disk (Rand 1996; Rossa \& Dettmar 2003a).

Previous studies on minor axis outflows in Seyfert galaxies have been performed mostly by $\mathrm{H} \alpha$ imaging (e.g., Pogge 1989; Colbert et al. 1996). However, only a few galaxies have been studied in [O III] in the past (e.g., Pogge 1988a,b, 1989; Veilleux et al. 1999a, 2003), and these were mostly relatively face-on galaxies. Apart from the recent study of NGC 4388 with the SUBARU telescope (Yoshida et al. 2002), most observations had limited sensitivity, and only nucleated minor axis outflows in $\mathrm{H} \alpha$ were detected. Furthermore, the extent of the regions around 
the galaxies studied was limited by detector size (i.e. limited field of view). By contrast, the recent deep narrowband imaging in the Seyfert galaxy NGC 4388 revealed extended emission from minor axis outflows up to distances of $|z| \sim 35 \mathrm{kpc}$. Previous detection rates of large scale minor axis outflows in edge-on Seyfert galaxies were found to be about 27\% (Colbert et al. 1996). More recently, Veilleux et al. (2003) presented results of a Taurus tunable filter study of 10, mostly face-on, starburst/AGN galaxies. Their overall detection rate was $60 \%$, but when only counting the six Seyfert galaxies in their sample, all of those showed large scale outflows.

The origin of very extended emission line regions is not quite clear yet. A likely origin for the extended emission in the Virgo Cluster galaxy NGC 4388 may be ram pressure stripping (Yoshida et al. 2004), as had been previously suggested for the peculiar gas morphology in another Virgo cluster spiral, NGC 4522 (Kenney \& Koopmann 1999; Kenney et al. 2004). Puzzling excitation structures of an outflow were reported for the galaxy NGC 1482, using tunable filters (Veilleux \& Rupke 2002), although on somewhat smaller scales than for the NGC 4388 case. For a general review on galactic winds we refer the reader to the recent paper by Veilleux et al. (2005).

In a recent investigation using $\mathrm{H} \alpha$ imaging of gaseous outflows in nearby edge-on galaxies it was shown that there are differences between starburst and non-starburst galaxies (Rossa \& Dettmar 2003a). This is manifested by their loci in a so-called diagnostic diagram for diffuse emission, based on the strength of star formation rate per unit area as a function of the far infrared flux ratio of 60 to 100 microns (which is a proxy of the warm dust temperature). Galaxies with higher star formation rate and warmer dust are more likely to show outflows. In the Rossa \& Dettmar (2003a) study, Seyfert galaxies were basically excluded. The present paper fills this gap by studying the gaseous outflows in a sample of Seyfert galaxies. We observed this sample both in $\mathrm{H} \alpha$ and [O III] emission. The excitation mechanisms at work can potentially be distinguished by the fact that the [O III] and $\mathrm{H} \alpha$ emission will have different responses to ionizing contributions of a hard power-law and from stellar radiation. Different excitation mechanisms might therefore be distinguishable using $\mathrm{H} \alpha$ to [O III] ratio maps.

The method of narrowband imaging has the advantage that excitation mechanisms can be studied in a much less time consuming manner than by longslit spectroscopy. The whole galaxy can be investigated in one single pointing per emission line. By contrast, longslit spectroscopy can only measure line ratios at specific cuts perpendicular or parallel to the major axis, where emission is already known from previous imaging. However, in many cases the presence or distribution of extended minor axis gaseous outflows in Seyfert galaxies is not known at all. A multifilter narrowband imaging study of a homogeneous sample of Seyfert galaxies is therefore a natural first step. With this approach we would like to investigate the role of the AGN versus the contribution to the IGM enrichment by disk outflows from strong $\mathrm{H}$ II regions. We can also study environmental effects such as past mergers (Walker et al. 1996) and ram pressure stripping (Cayatte et al. 1994), by considering how disturbed the extended emission line regions are.

The paper is structured in the following way. In Sect. 2 we present the sample, describe the observations and present the data reduction techniques. In Sect. 3 we present the results for each individual galaxy, including a basic description of the observed morphology as seen in the broadband (e.g., $R$ band) and narrowband ( $\mathrm{H} \alpha$ and [O III]) images. In Sect. 4 we discuss the results in terms of excitation maps and we attempt to discriminate between minor axis nuclear and disk outflows. Finally, in Sect. 5 we present our summary.

\section{Sample, observations and data reduction}

\subsection{The Seyfert galaxy sample}

Our sample is a distance-limited sample of nearby edge-on Seyfert galaxies. We selected the galaxies according to the following criteria: all targets have radial velocities of $v_{\text {rad }} \leq$ $5300 \mathrm{~km} \mathrm{~s}^{-1}$, and inclinations of $i \geq 80^{\circ}$. The first selection criterion assures that sufficiently high spatial resolution is reached in order to resolve individual emission features, such as filaments. The galaxies in our sample have distances of $10.6 \mathrm{Mpc} \leq D \leq 73.0 \mathrm{Mpc}$, assuming a Hubble constant of $H_{0}=75 \mathrm{~km} \mathrm{~s}^{-1} \mathrm{Mpc}^{-1}$. This translates to spatial scales that can be resolved in the range of $50 \mathrm{pc}$ to $350 \mathrm{pc}$ for our targets. The inclination criterion is essential for detections of minor axis outflows, since in less inclined targets extended emission cannot be distinguished unambiguously from disk emission.

The resulting sample consists of 14 northern hemisphere edge-on Seyfert galaxies that were selected from the electronic version of the 10th edition of A Catalogue of Quasars and Active Nuclei, published by Véron-Cetty \& Véron (2001). We have chosen only galaxies classified as being Seyfert (type 1 and 2) galaxies, thus rejecting H II galaxies and LINERs. All relevant galaxies were initially inspected visually using Digitized Sky Survey (DSS) images to verify their edge-on orientation prior to making it into our final list. Our selection criteria are not too different from the $\mathrm{H} \alpha$ imaging study by Colbert et al. (1996). However, we found that several of the galaxies previously studied by Colbert et al. (1996) were far from being archetypical edge-on galaxies. In addition we include some known Seyferts, based on the search in Véron-Cetty \& Véron (2001) that are not constrained in the Colbert et al. (1996) study. The selected sample consisted of 30 galaxies, visible from both hemispheres. We only observed the 14 galaxies visible from the northern hemisphere. In Table 1 we list the basic parameters of these galaxies, such as coordinates, sizes, distances and magnitudes.

The advantage of using a distance-limited sample is that the galaxies can be studied with high angular resolution and sensitivity. It has the disadvantage that only a relatively small volume is probed. Since the most luminous AGN are rare, our sample is necessarily restricted to "low-luminosity" Seyfert galaxies. Our study can therefore only make statements about extended emission-line gas and minor-axis outflows in Seyfert galaxies in this luminosity regime. The results cannot necessarily be generalized to higher-luminosity Seyfert galaxies, since it is likely that more luminous AGN are able to drive stronger outflows. Our study is therefore complementary to possible studies of more distant high-luminosity Seyferts. Such studies would have different goals and limitations. For example, there would be less spatial resolution and thus less sensitivity to detection of narrow features (e.g., filaments). Moreover, the highest-luminosity AGN often have ongoing star-formation, or are involved in a galaxy-galaxy interaction. This makes it more difficult to establish whether any outflow is driven by the AGN or by the starburst.

\subsection{Observations}

The sample was imaged in two observing runs. Optical $B$ and $R$-band images, and narrowband $\mathrm{H} \alpha$ and [O III] images were taken in the periods from October 28th to 30th 2002 and 
Table 1. Basic properties of the galaxy sample.

\begin{tabular}{lccccccc}
\hline \hline Galaxy & $\begin{array}{c}\text { RA (J2000.0) } \\
{[\text { hh mm ss.s }]} \\
(2)\end{array}$ & $\begin{array}{c}\text { Dec (J2000.0) } \\
{\left[{ }^{\circ} \prime^{\prime \prime}\right]} \\
(3)\end{array}$ & $\begin{array}{c}c z \\
{\left[\mathrm{~km} \mathrm{~s}^{-1}\right]} \\
(4)\end{array}$ & $\begin{array}{c}\text { Distance } \\
{[\mathrm{Mpc}]} \\
(5)\end{array}$ & $\begin{array}{c}a \times b \\
{\left[{ }^{\prime} \times{ }^{\prime}\right]} \\
(6)\end{array}$ & $\begin{array}{c}m_{B} \\
{[\mathrm{mag}]} \\
(7)\end{array}$ & $\begin{array}{c}L_{\mathrm{FIR}} \\
{\left[\mathrm{erg} \mathrm{s}^{-1}\right]} \\
(8)\end{array}$ \\
\hline Mrk 993 & 012531.4 & +320811 & 4658 & 62.1 & $2.2 \times 0.7$ & 13.96 & $2.78 \times 10^{43}$ \\
Mrk 577 & 014929.9 & +123032 & $5221^{a}$ & 69.6 & $1.3 \times 0.5$ & 14.10 & $\ldots$ \\
UGC 1479 & 02 00 19.1 & +242825 & $4927^{a}$ & 65.7 & $1.3 \times 0.4$ & 14.90 & $1.00 \times 10^{44}$ \\
Ark 79 & 02 17 23.0 & +382450 & $5254^{a}$ & 70.1 & $0.8 \times 0.2$ & 13.84 & $\ldots$ \\
Mrk 1040 & 02 28 14.6 & +311841 & 4993 & 66.6 & $3.9 \times 0.8$ & 14.74 & $1.66 \times 10^{44}$ \\
UGC 2936 & 04 02 48.3 & +015757 & 3813 & 50.8 & $2.5 \times 0.7$ & 15.00 & $1.98 \times 10^{44}$ \\
NGC 3079 & 100157.8 & +554047 & 1125 & 15.0 & $7.9 \times 1.4$ & 11.43 & $1.39 \times 10^{44}$ \\
NGC 3735 & 113557.3 & +703209 & 2692 & 35.9 & $4.2 \times 0.8$ & 12.57 & $1.46 \times 10^{44}$ \\
NGC 4235 & 121709.9 & +071129 & 2410 & 32.1 & $4.2 \times 0.9$ & 12.64 & $4.67 \times 10^{42}$ \\
NGC 4388 & 122546.7 & +123944 & 2517 & 33.6 & $4.8 \times 0.9$ & 11.91 & $1.61 \times 10^{44}$ \\
NGC 4565 & 123620.8 & +255916 & 1282 & 17.1 & $15.9 \times 1.9$ & 10.18 & $3.71 \times 10^{43}$ \\
NGC 5866 & 150629.6 & +554548 & $769^{a}$ & 10.3 & $4.7 \times 1.9$ & 11.26 & $9.97 \times 10^{42}$ \\
IC 1368 & 211413.0 & +021041 & 3912 & 52.2 & $1.1 \times 0.4$ & 14.30 & $1.42 \times 10^{44}$ \\
UGC 12282 & 225855.5 & +405553 & 5094 & 67.9 & $1.9 \times 0.5$ & 14.70 & $8.06 \times 10^{43}$ \\
\hline
\end{tabular}

Notes: column (1) lists the galaxy name. Columns (2) and (3) list the coordinates taken from the NASA Extragalactic Database (NED). Column (4) gives the radial velocity, measured from H I data and taken from NED. Data labeled with " $a$ " refer to velocity data from optical measurements. Column (5) gives the corresponding distances assuming $H_{0}=75 \mathrm{~km} \mathrm{~s}^{-1} \mathrm{Mpc}^{-1}$. Column (6) lists the optical extent of the major and minor axis taken from NED. Column (7) lists the $B$-band magnitude, also taken from NED. Finally, in Col. (8) we list the FIR luminosity, calculated from $F_{60}$ and $F_{100}$ (taken from NED) using the equation in Rossa \& Dettmar (2000). For Ark 79 and Mrk 577 there are no IRAS fluxes available, therefore $L_{\mathrm{FIR}}$ was not computed.

from April 5th to 6th 2003. We used the Bonn University Simultaneous Camera (BUSCA), attached to the $2.2 \mathrm{~m}$ telescope at the Calar Alto Observatory. BUSCA (Reif et al. 1999) is a four-channel CCD camera which allows simultaneous imaging of a $12^{\prime} \times 12^{\prime}$ field of view in four different wavebands, each of which are separated by dichroic filters. The wavelength range covered by these four CCD cameras is $\lambda \leq 4300 \AA$ (UV), 4300 to $5400 \AA$ (Blue), 5400 to $7300 \AA$ (Red), and $\lambda \geq 7300 \AA$ (NIR). Three of the four CCDs are frontsize devices (CCD485 Lockheed Martin), with a pixel array of $4096 \times 4096$ pixels, and with a pixel size $15 \mu \mathrm{m} \times 15 \mu \mathrm{m}$. The CCD for the UV channel is a backside thinned CCD485. The pixel scale in the $2 \times 2$ binning configuration used was 0!35. Further details on BUSCA can be found at the BUSCA webpage ${ }^{1}$.

We used the $\mathrm{H} \alpha$ and [O III] filters for channel 2 and 3, whereas channel 1 and 4 were equipped with a UV and $I$-band filter. The broadband images used for our analysis were instead obtained in channel 2 and 3, subsequently, since their bandpasses are located in the same wavelength region as the narrowband filters. To avoid possible excessive straylight (Oliver Cordes for the BUSCA team, priv. comm.), channels 2 and 3 were not equipped with actual $B$ and $R$ broadband filters. Instead, the dichroic filters already on the instrument adequately mimic the transmission of the standard $B$ and $R$-bands. The transmission curves of these dichroic filters are shown in Fig. 1. Throughout this paper we speak of $B$ - and $R$-band images, when we refer to images obtained with these dichroic filters. Details on the narrowband filter specifications such as central wavelength and FWHM are listed in Table 2, which also presents a log of the observations that includes observation dates, exposure times, and seeing values. Due to the $F W H M$ of the $\mathrm{H} \alpha$ filters, emission from the adjacent [N II] doublet is also included in the bandpass. For the remainder of the paper we always refer for simplicity to images obtained with these filters as $\mathrm{H} \alpha$ images.

\footnotetext{
${ }^{1}$ http://www. caha.es/guijarro/BUSCA/busca.html
}

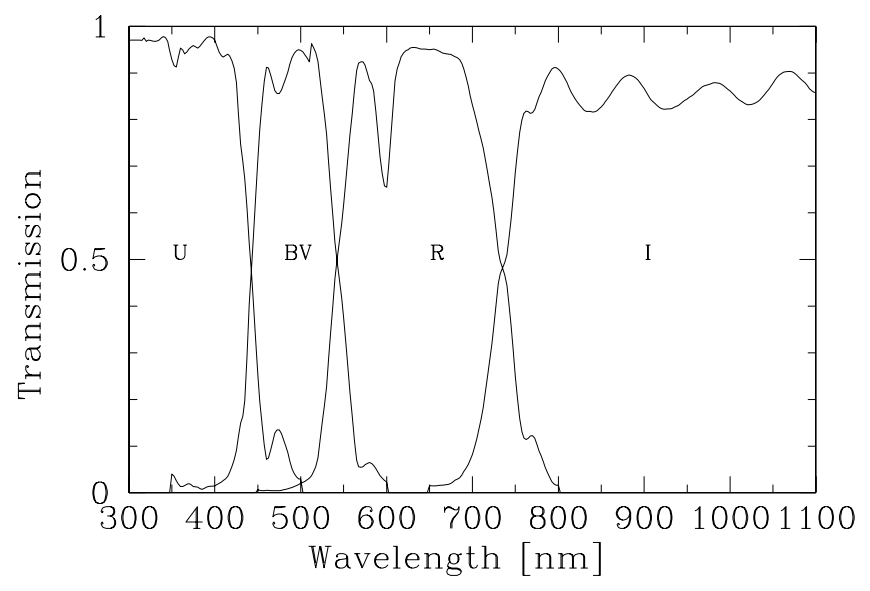

Fig. 1. Transmission curves for the four dichroic filters of the BUSCA instrument. The positions in wavelength of standard broadband filters are indicated.

\subsection{Data analysis}

\subsubsection{Data reduction}

The data reduction was performed in the usual way using the $\mathrm{IRAF}^{2}$ ccdproc package. A pedestal count level was determined and subtracted from each image using the overscan region. Ten to twenty bias frames were combined using the zerocombine task to create one master bias for each night and each of the four channels. The appropriate bias frames were subtracted from the flat-field frames and images to remove any twodimensional structure remaining after the pedestal level subtraction. However, this bias subtraction was only performed on the $\mathrm{H} \alpha$ and $R$-band images. There were variability problems in the

\footnotetext{
${ }^{2}$ IRAF is distributed by the National Optical Astronomy Observatory, which is operated by the Association of Universities for Research in Astronomy, Inc. (AURA) under cooperative agreement with the National Science Foundation.
} 


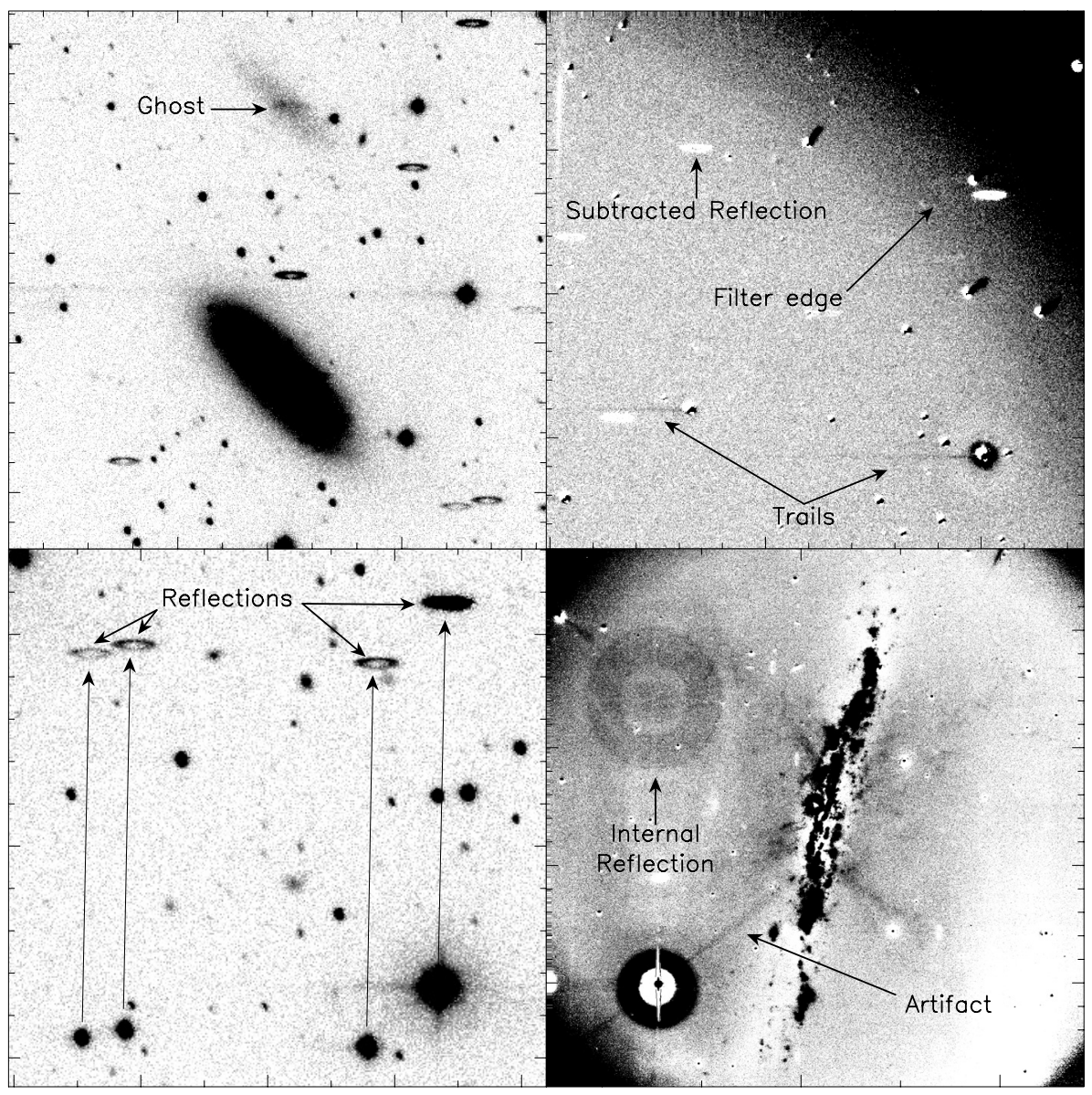

Fig. 2. Gallery of the various remaining image artifacts found in the BUSCA data. A detailed description of these artifacts is given in Sect. 2.3.2. The images show some of the "worst case" examples. With proper care in the analysis our study was not generally affected by any of these artifacts. bias images from the $\mathrm{CCD}$ used for the [O III] and $B$-band images. Since the field-dependent variations of the bias is small, we decided not to use these images, and stick with a single constant pedestal instead. The flatfields were combined in groups of three to eight frames to give one normalized master flatfield per night, channel, and filter. These were used to flatfield the data.

The sky background in the images was determined by manually selecting in each frame three regions which showed no contamination from the galaxy or from any stars, and measuring the median sky value. The three values were then averaged to give a final sky background value. If the scatter in the three values was above a threshold of five counts, the regions used to calculate the medians were changed. The sky background thus determined was subtracted. We estimate the uncertainties in the background values to be less than a few percent. In the next step the images were aligned by performing a cross-correlation using the xregister task. First, a Fourier cross-correlation was used to correct for large shifts with a nearest pixel interpolation, then a finer discrete cross-correlation was used, applying a polynomial interpolation. The image frames were then combined using the imcombine task. Since at least three narrowband frames were available for most of our galaxies, a median combination was sufficient to eliminate the cosmic rays from the narrowband observations. For the broadband observations, only two frames were acquired for most galaxies. These were combined using an average combination along with the crreject rejection criterion to eliminate the majority of the cosmic ray events. In one specific case (NGC 4388) only one frame was obtained in all filters, and the cosmicrays package was used in order to eliminate the cosmic rays.
Once cosmic-ray free images were obtained, the images from the different filterbands for a specific galaxy were aligned. This is a requirement for the continuum subtraction, which involves a scaling of different filter images. The alignment involved a small rotation, as the different channels did not have the exact same orientation (the measured difference was typically less than $1^{\circ}$ ). A polynomial interpolation was used to accommodate the difference in rotation angle. The images were then aligned using a discrete-cross correlation, once again applying a polynomial interpolation.

\subsubsection{Remaining data artifacts}

Besides the well known detector artifacts (e.g., those produced by cosmic-ray events), which can be corrected for by combining individual images, there are also instrumental artifacts caused by the optical system. This may leave additional artifacts (e.g., reflections) that we discuss in the following. Due to the optical outline of the BUSCA instrument (involving dichroic beamsplitters) in conjunction with the optical assembly of the telescope, there are several artifacts present in our images. We address these artifacts here and show examples in Fig. 2.

In all broadband images there are internal reflections from bright and moderately bright point sources (i.e. stars), which appear as slightly flattened, oval disks, with a central cavity (see upper left panel of Fig. 2). These reflections always have a constant distance from the source and appear $\approx 90^{\prime \prime}$ to the $\mathrm{N}$ of the stars in the $B$-band, and also appear $\approx 90^{\prime \prime}$ to the $\mathrm{W}$ and to the $\mathrm{N}$ in the $R$-band. The reflections are brightest in the $B$-band images. Since these reflections have a constant distance from their source (see lower left panel of Fig. 2), they are easy to 

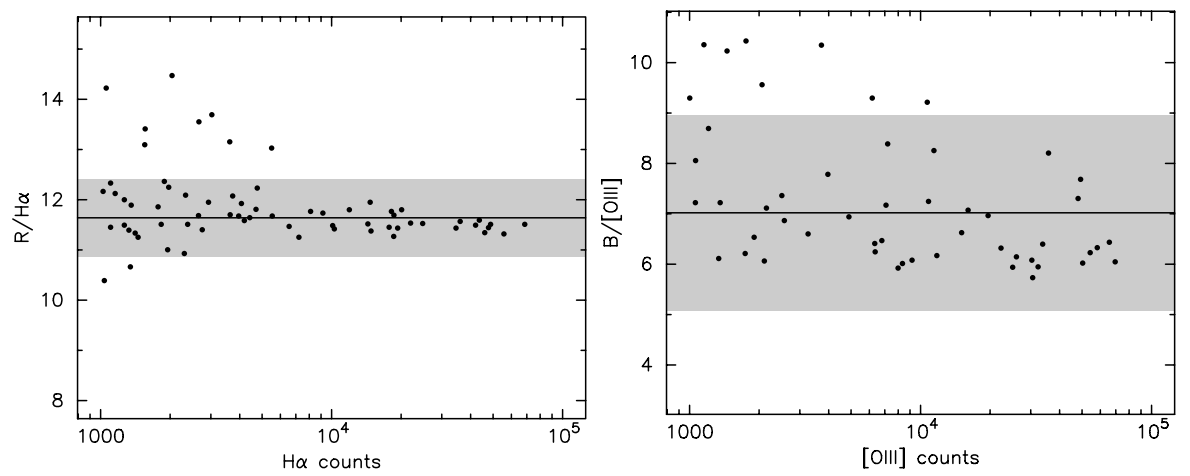

Fig. 3. Broadband to narrowband ratios of the foreground stars in the field of view of UGC 12282. The shaded region marks the $1 \sigma$ scatter. The $\mathrm{R} / \mathrm{H} \alpha$ ratio is shown in the left panel, whereas the $\mathrm{B} /[\mathrm{O} \mathrm{III}]$ ratio is shown in the right panel. The ratios are in counts with exposure times scaled to correspond to the Ark 79 values. identify and thus cannot be mistaken for any of the diffuse structures we are interested in. Furthermore, in the continuum subtracted images they appear as white oval disks (see upper right panel of Fig. 2) in contrast to the true galaxy features, which are shown in black in our "inverted" grey-scale images (see lower right panel of Fig. 2). Only in one case (UGC 1479), was such an artifact directly superposed onto the southern part of the galaxy disk. Bright extended sources such as the galaxy bulge of course also produce ghost images at the same distance and orientation (N of the disk) in the $B$-band images (see upper left panel of Fig. 2). But because of the $90^{\prime \prime}$ offset, our analysis at the galaxies themselves is not affected by this. Other internal reflections around unusually bright stars in the field are also visible (see lower right panel of Fig. 2), reminiscent of the bright reflections seen in some of the Digitized Sky Survey images which have bright stars in the field. We have obviously taken extreme care not to mistake any of the associated diffraction spikes with emission filaments. Another artifact, affecting all CCDs to some extent, are the trails seen emerging from bright stars (see upper right panel of Fig. 2). These are so called charge transfer inefficiencies.

It should also be noted that the narrowband filters obstructed the extreme outer boundaries of the field of view (see upper right panel of Fig. 2). This has no effects on any of our science cases, as most galaxies are much smaller than the field of view.

\subsubsection{Narrowband emission maps}

In order to generate continuum-free $\mathrm{H} \alpha$ and [O III] images, the broadband images were appropriately scaled and subtracted from the narrowband images. The $R$-band images were scaled and subtracted from the $\mathrm{H} \alpha$ images, and the $B$-band images were scaled and subtracted from the [O III] images. The scale factors were determined empirically.

In order to determine the $R$-band to $\mathrm{H} \alpha$ and $B$-band to [O III] ratios, we first performed aperture photometry on stars in both frames using the apphot package, attempting to include as many true stellar point sources as possible, while excluding background galaxies. Figure 3 shows the values of the ratio obtained for all the stars in the UGC 12282 field. This field has the largest number of foreground stars, and therefore the ratios were best determined for this galaxy. Since some of the objects included in the photometry may still be non-stellar sources (e.g., faint background galaxies), we used the median ratio rather than the mean as an indicator of the overall ratio. The scatter at low count values is likely to be due to Poisson noise or in some cases non-stellar sources, but overall the $R$ to $\mathrm{H} \alpha$ ratio does seem well constrained. Unfortunately, the $R$ to $\mathrm{H} \alpha$ ratio was not as well constrained for most of the other galaxies, due to contamination from background galaxies and the lack of sufficient numbers of suitable stars in many of the frames. The $B$ to [O III] ratio was not tightly constrained in any of the galaxies. Either way, the average color of foreground stars is probably not representative of the galaxies that we are interested in. This might bias the determination of the scale factors. So we decided to use a different approach instead, and to use aperture photometry results only as a consistency check.

We ultimately defined the broadband to narrowband ratio to be the ratio that minimizes the number of pixels with narrowband emission, whilst also minimizing the number of oversubtracted pixels. In practice this was done by selecting a patch of background sky and finding the standard deviation of the pixel counts within this region. We then considered all the pixels within a circular region, centered on each galaxy, and varied the broadband to narrowband ratio until the number of pixels with count values within one standard deviation from the mean background value was maximized. In order to verify this method we compared the ratio obtained to the method described above. We found the $R$ to $\mathrm{H} \alpha$ ratio for UGC 12282 to be 11.23 using the new algorithm, compared to $11.64 \pm 0.77$ for the initial method, which is in reasonable agreement. The $B$ to $[\mathrm{O} \mathrm{III}]$ ratio was found to be 6.94 using the new algorithm, compared to $7.02 \pm 1.94$ for the initial method, once again in good agreement.

The final ratios used for each galaxy to construct the $\mathrm{H} \alpha$ and [O III] maps are shown in Fig. 4, and are normalized to the individual exposure times. In the case of the $R / \mathrm{H} \alpha$ ratio, the values for the NGC galaxies are smaller than for the other galaxies. This agrees with the fact that the overall transmission of the filter used for the NGC galaxies is approximately $10 \%$ larger than the transmission of the filter used for the more remote galaxies. Reversely, the larger $B /[\mathrm{O} \mathrm{III}]$ ratio for the NGC galaxies compared to the other galaxies is due to the fact that the [O III] filter used for the NGC galaxies has a transmission approximately $30 \%$ less than for the other galaxies. The relatively small variations in the ratio from galaxy to galaxy (observed with the same filter) confirm the accuracy of the method. Some of the remaining scatter may be due to the fact that the observing conditions were not photometric.

\subsubsection{Flux calibration}

The observations were partially carried out under nonphotometric conditions. Therefore, no standards were acquired in the broadband channels. A flux calibration was performed using a narrowband image containing three calibration stars. The stars used were PG 0231+051, PG 0231+051A and PG0231+051D, and the standard magnitudes were taken from Landolt (1992). The predicted flux of the stars in $\mathrm{H} \alpha$ and 

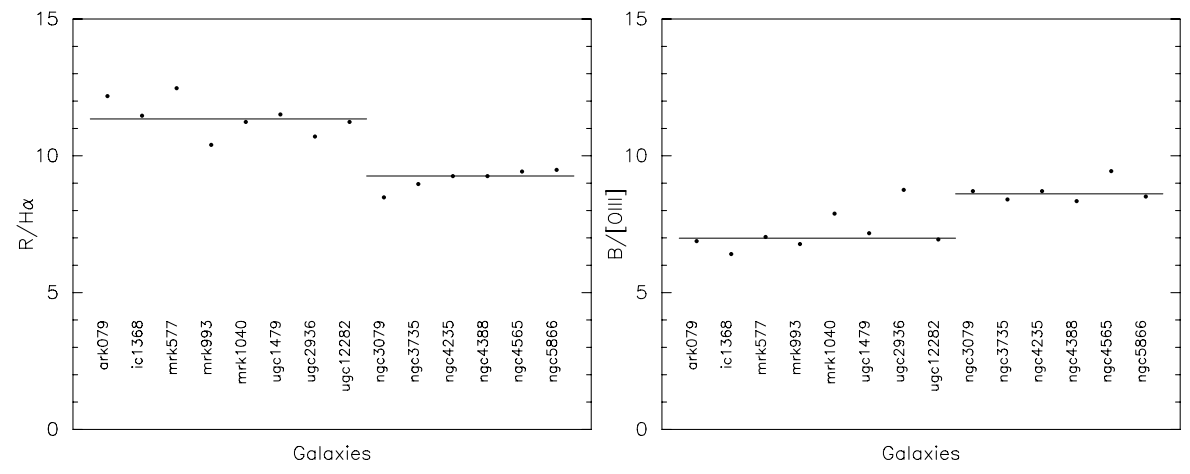

Fig. 4. Broadband to narrowband ratios for all galaxies in $\mathrm{H} \alpha($ left $)$ and [O III] (right). The thin lines shows the median of the ratio for the nonNGC galaxies, observed in the first run, and the median of the ratio for the NGC galaxies, observed in the second run with a different filter. The ratios are in counts with exposure times scaled to correspond to the Ark 79 values.
[O III] was found by normalizing a stellar spectrum to each of the known $B$-, $R$-, and $I$-band magnitudes in turn, each time extrapolating the flux to the $\mathrm{H} \alpha$ and [O III] wavelengths. The results using the three different broadband magnitudes were consistent with each other, and the calibration factors obtained for each of the three stars were also consistent. After taking the filter transmission into account, we found the calibration factors to be $(1.06 \pm 0.06) \times 10^{-15} \mathrm{erg} \mathrm{cm}^{-2}$ count $^{-1}$ for the H $\alpha$ images and $(1.01 \pm 0.06) \times 10^{-15} \mathrm{erg} \mathrm{cm}^{-2}$ count $^{-1}$ for the $[\mathrm{O} \mathrm{III]}$ images. The errors represent the standard deviation of the values for the three stars and do not account for potential systematics.

The $\mathrm{H} \alpha$ and [O III] emission we observe from the various galaxies only spans a narrow range of wavelengths compared to the width of the filters. On the other hand, the flux from the calibration star was continuum emission, and therefore filled the whole filter. Therefore, before applying the calibration factor to the emission maps, we corrected for the filter transmission at the expected wavelength of the emission lines of the various galaxies. The expected wavelength of the $\mathrm{H} \alpha$ and [O III] lines were found by using the recession velocities of the galaxies. The [O III] emission line is actually a doublet, which means that the correction factor was found by taking the two lines into account (we assumed the $\lambda 4959 /[\mathrm{O}$ III] $\lambda 5007$ ratio to be $1 / 3$ ).

The total $\mathrm{H} \alpha$ and [O III] flux of each galaxy was determined by summing up the positive counts in a rectangular aperture enclosing the galaxy. We subtracted any remaining contributions from stars enclosed in the aperture. The $\mathrm{H} \alpha$ and [O III] fluxes for our sample are presented in Table 3 . To check our calibration and continuum subtraction method, we compared our $\mathrm{H} \alpha$ fluxes for five galaxies with previously published values (Colbert et al. 1996; Lehnert \& Heckman 1996b). The comparison is summarized in Table 4. The published $\mathrm{H} \alpha$ luminosities are in fairly good agreement with our values, with on average $\log \left(L_{\mathrm{us}} / L_{\mathrm{lit}}\right)=$ $-0.12 \pm 0.09$. This is probably a fair assessment of the accuracy of our absolute flux calibration, including both random and systematic errors. We did not find any published [O III] luminosities for any of the galaxies in our sample. However, the absolute flux calibration of the $\mathrm{H} \alpha$ and [O III] data was carried out in an analogous fashion. So we expect the uncertainties in both to be comparable.

\subsubsection{Detection sensitivity}

To calculate the sensitivity of our observations for detection of faint low surface brightness emission we quantified the noise in the final emission-line maps. We did this using two separate methods.
Table 3. $\mathrm{H} \alpha$ and [O III] fluxes and luminosities of the galaxies.

\begin{tabular}{lcccc}
\hline \hline Galaxy & $\begin{array}{c}F(\mathrm{H} \alpha) \\
{\left[\mathrm{erg} \mathrm{s}^{-1} \mathrm{~cm}^{-2}\right]} \\
(2)\end{array}$ & $\begin{array}{c}L(\mathrm{H} \alpha) \\
{[\log L]} \\
(3)\end{array}$ & $\begin{array}{c}F([\mathrm{OIII}]) \\
{\left[\mathrm{erg} \mathrm{s}^{-1} \mathrm{~cm}^{-2}\right]} \\
(4)\end{array}$ & $\begin{array}{c}L([\mathrm{OIII}]) \\
{[\log L]} \\
(5)\end{array}$ \\
\hline Mrk 993 & $1.04 \times 10^{-13}$ & 40.68 & $4.84 \times 10^{-14}$ & 40.35 \\
Mrk 577 & $4.19 \times 10^{-14}$ & 40.39 & $3.70 \times 10^{-14}$ & 40.33 \\
UGC 1479 & $8.23 \times 10^{-14}$ & 40.63 & $3.47 \times 10^{-14}$ & 40.26 \\
Ark 79 & $1.67 \times 10^{-13}$ & 40.99 & $1.22 \times 10^{-13}$ & 40.86 \\
Mrk 1040 & $6.82 \times 10^{-13}$ & 41.56 & $1.97 \times 10^{-13}$ & 41.02 \\
UGC 2936 & $1.25 \times 10^{-13}$ & 40.59 & $2.96 \times 10^{-14}$ & 39.96 \\
NGC 3079 & $2.94 \times 10^{-12}$ & 40.90 & $1.23 \times 10^{-12}$ & 40.52 \\
NGC 3735 & $9.04 \times 10^{-13}$ & 41.15 & $2.47 \times 10^{-13}$ & 40.58 \\
NGC 4235 & $1.26 \times 10^{-13}$ & 40.20 & $6.25 \times 10^{-14}$ & 39.89 \\
NGC 4388 & $2.22 \times 10^{-12}$ & 41.48 & $1.59 \times 10^{-12}$ & 41.33 \\
NGC 4565 & $1.24 \times 10^{-12}$ & 40.64 & $8.35 \times 10^{-13}$ & 40.47 \\
NGC 5866 & $\ldots$ & $\ldots$ & $\ldots$ & $\ldots$ \\
IC 1368 & $9.04 \times 10^{-14}$ & 40.47 & $2.34 \times 10^{-14}$ & 39.88 \\
UGC 12282 & $4.18 \times 10^{-14}$ & 40.37 & $1.83 \times 10^{-14}$ & 40.01 \\
\hline
\end{tabular}

Notes: column (1) lists the galaxy name. Column (2) lists the total $\mathrm{H} \alpha$ flux and Col. (3) lists the corresponding luminosity, assuming the distances quoted in Table 1. Similarly, Cols. (4) and (5) list the total [O III] fluxes and luminosities. For NGC 5866 no accurate flux measurements were possible, so we did not list any.

Table 4. Comparison of $\mathrm{H} \alpha$ luminosities with literature values.

\begin{tabular}{lcc}
\hline \hline $\begin{array}{l}\text { Galaxy } \\
(1)\end{array}$ & $\begin{array}{c}\log L(\mathrm{H} \alpha) \\
(2)\end{array}$ & $\begin{array}{c}\log L_{\mathrm{lit}}(\mathrm{H} \alpha) \\
(3)\end{array}$ \\
\hline Mrk 993 & 40.68 & $40.87^{a}$ \\
Ark 79 & 40.99 & $40.95^{a}$ \\
NGC 3079 & 40.90 & $41.06^{b}$ \\
NGC 4235 & 40.20 & $40.38^{a}$ \\
IC 1368 & 40.47 & $40.56^{a}$ \\
\hline
\end{tabular}

Notes: column (1) lists the galaxy name. Column (2) lists our total $\mathrm{H} \alpha$ luminosity and Col. (3) lists the total $\mathrm{H} \alpha$ luminosity quoted in the literature. ${ }^{a}$ Data taken from Colbert et al. (1996), ${ }^{b}$ data taken from Lehnert \& Heckman (1996b).

In the first method we calculated the noise on the basis of statistical considerations, using the known sky background, detector properties and observational setup. The values of the sky background computed for the sky subtraction (see Sect. 2.3.1) were converted from counts to electrons using the gain values listed on the BUSCA webpage (see footnote 1 ); i.e., $1.2136 \mathrm{e}^{-} /$count for the $B$ and [O III] images, and $2.9674 \mathrm{e}^{-} /$count for the $R$ and $\mathrm{H} \alpha$ images. The result was used to estimate the contribution to the noise from Poisson statistics in 
the final broadband and narrowband images. To this we added in quadrature the contribution from read-noise, taking into account the number of exposures. The detector read-noise values are also given on the BUSCA webpage; i.e., $6.37 \mathrm{e}^{-}$for the $B$ and [O III] images, and $7.60 \mathrm{e}^{-}$for the $R$ and $\mathrm{H} \alpha$ images. The results were used to estimate the 1- $\sigma$ noise level in electrons per pixel in each final continuum-subtracted emission-line image. These were used to calculate the noise in electrons per square arcsec, using the known pixel size and the fact that noise from co-added independent pixels adds in quadrature. The results were transformed to units of $\mathrm{erg} \mathrm{s}^{-1} \mathrm{~cm}^{-2} \operatorname{arcsec}^{-2}$ using the detector gain values and the flux calibrations described in Sect. 2.3.4. The predicted $1-\sigma$ noise values for the $\mathrm{H} \alpha$ and [O III] emission-line maps are listed in Table 5.

In the second method for estimating the noise in the emission-line maps we used the histogram of the pixel values in an "empty" region of each emission-line map (i.e., away from the galaxy, bright stars, ghosts, and detector artifacts). We measured the FWHM and divided by 2.355 to obtain an estimate of the 1- $\sigma$ noise level in counts per pixel (a Gaussian distribution has $\sigma=F W H M / 2.355$; basing the calculation on the $F W H M$ value rather than the second moment has the advantage of robustness against outlier pixels). This was transformed to units of $\operatorname{erg~s}^{-1} \mathrm{~cm}^{-2} \operatorname{arcsec}^{-2}$ in the same manner as for the first method. The measured 1- $\sigma$ noise values for the $\mathrm{H} \alpha$ and [O III] emissionline maps are also listed in Table 5. The second method has the advantage that it takes into account potential additional sources of noise that cannot be accounted for by idealized statistical calculations (e.g., flat-fielding errors). However, the predicted and measured values are overall in very good agreement, which gives extra confidence in the results.

The $\mathrm{H} \alpha$ and $[\mathrm{O} \mathrm{III}]$ narrow-band observations contribute roughly twice as much noise to the final emission-line maps as the scaled $R$ and $B$-band images. The main contributor to the noise in the $\mathrm{H} \alpha$ and [O III] narrow-band images is the Poisson noise from the background, which is on average two to four times as large as the contribution from the readout noise. The galaxy with the lowest predicted and measured noise level in both the $\mathrm{H} \alpha$ and [O III] emission line maps is UGC 12282, with a predicted $1-\sigma$ noise level of $8.56 \times 10^{-18} \mathrm{erg} \mathrm{s}^{-1} \mathrm{~cm}^{-2} \operatorname{arcsec}^{-2}$ in $\mathrm{H} \alpha$ and $1.59 \times 10^{-17} \mathrm{erg} \mathrm{s}^{-1} \mathrm{~cm}^{-2} \operatorname{arcsec}^{-2}$ in [O III]. The galaxy with the highest noise level is NGC 4388, since for this galaxy only one frame was available in each filter. The predicted 1- $\sigma$ noise levels for this galaxy are $3.10 \times 10^{-17} \mathrm{erg} \mathrm{s}^{-1} \mathrm{~cm}^{-2} \operatorname{arcsec}^{-2}$ in $\mathrm{H} \alpha$ and $4.43 \times 10^{-17} \mathrm{erg} \mathrm{s}^{-1} \mathrm{~cm}^{-2} \operatorname{arcsec}^{-2}$ in [O III].

We take three times the predicted $1-\sigma$ noise level for each emission line map as a measure of our final sensitivity for detection of faint low surface brightness emission. The 3- $\sigma$ detection sensitivities thus obtained for each galaxy are also listed in Table 5. The median sensitivity over the sample is $3.6 \times 10^{-17} \mathrm{erg} \mathrm{s}^{-1} \mathrm{~cm}^{-2} \operatorname{arcsec}^{-2}$ for the $\mathrm{H} \alpha$ images and $6.9 \times 10^{-17} \mathrm{erg} \mathrm{s}^{-1} \mathrm{~cm}^{-2} \operatorname{arcsec}^{-2}$ for the [O III] images. The $\mathrm{H} \alpha$ intensity $I$ can be directly transformed into a line emission measure (EM), which is defined as the line-of-sight integral over the squared electron density. For an ionized region that is thick to Lyman lines (case B recombination) one has (Reynolds 1992) that

$$
\frac{E M}{\mathrm{~cm}^{-6} \mathrm{pc}} \approx\left(\frac{I}{2.0 \times 10^{-18} \mathrm{erg} \mathrm{s}^{-1} \mathrm{~cm}^{-2} \operatorname{arcsec}^{-2}}\right)\left(\frac{T}{10^{4} \mathrm{~K}}\right)^{-0.92}
$$

So if we assume an electron temperature $T \approx 8000 \mathrm{~K}$ as in Veilleux et al. (1995), then our median sensitivity limit for $\mathrm{H} \alpha$ corresponds to $22 \mathrm{~cm}^{-6} \mathrm{pc}$.
The quoted sensitivities refer to detection over an area of 1 square arcsec. If one averages over larger areas then one can potentially see fainter emission, but there will be less information on spatial detail. Point-source sensitivities (which are of less interest in the context of the present paper) are similar to the values listed in Table 5, given that the seeing FWHM of most observations was close to 1 arcsec (see Table 2). The quoted limits do not take into account the noise and subtraction problems associated with galaxy continuum light. Given the edge-on viewing of the galaxies, the limits therefore apply to high-latitude emissionline gas only.

Our sensitivity limits are comparable to those determined for NGC 3079 by Veilleux et al. (1994, 1995), who quote $\sim 3 \times$ $10^{-17} \mathrm{erg} \mathrm{s}^{-1} \mathrm{~cm}^{-2} \operatorname{arcsec}^{-2}$. Our sensitivities are also similar to those achieved in the study of 9 galaxies by Rand (1996), who obtained observations with a comparable instrumental setup. Of course, pointed studies of individual galaxies with $8 \mathrm{~m}$-class telescopes (e.g., Yoshida et al. 2002) or detailed Fabry-Perot studies on 4 m-class telescopes (e.g., Veilleux et al. 2003) can achieve higher sensitivities than those obtained here. For comparison, for NGC 4388 our observations are a factor of four and ten ([O III $]$ and $\mathrm{H} \alpha$, respectively) less sensitive than the very deep SUBARU observations by Yoshida et al. (2002). (But our $\mathrm{H} \alpha$ sensitivity for this galaxy is low by a factor 2.6 relative to the median for the other galaxies in our own sample, due to the fact that we had only one exposure available.)

\subsubsection{The ionization maps}

Finally, $[\mathrm{O} \mathrm{III}] / \mathrm{H} \alpha$ ionization maps were constructed using the following procedure. First, the average remaining background value was subtracted from each $\mathrm{H} \alpha$ and [O III] map. This was done since in some cases the background value was not exactly zero, due to the process of combining the broadband and narrowband frames. We then performed a basic $1 \sigma$ clipping, by setting all pixels with count values lower than $1 \sigma$ of the background to zero. This included any negative count values. This was necessary in order to avoid divisions by very small numbers found in noise, which if divided by, would lead to values of the $[\mathrm{O}$ III $] / \mathrm{H} \alpha$ ratio larger than the actual signal itself. Following this, we divided the [O III] image by the $\mathrm{H} \alpha$ image in order to obtain the final ionization maps. We then applied a $3 \times 3$ median box filter to the ionization maps in order to reduce the noise. This approach is similar to the method used by Pogge (1988a). In Sect. 4.1 we discuss the results of the ionization maps, presented in Sect. 3.

\section{Results}

In the following descriptions, we quote angular separations and sizes of interest, and in each case we quote in brackets the corresponding projected physical sizes, assuming the distances given in Table 1 .

\subsection{Mrk 993}

Mrk 993 (Fig. 5) appears to be a relatively featureless spiral in the $R$-band. The $\mathrm{H} \alpha$ map shows emission from the nucleus, H II regions scattered throughout the disk (as previously observed by Colbert et al. 1996), and traces of diffuse emission between the compact emission regions. The H II regions are visible out to 53" (16.0 kpc) to the NE and 64" (19.3 kpc) to the SW of the nucleus. Also visible is an $\mathrm{H} \alpha$ emitting ring-like structure of radius $\sim 14^{\prime \prime}$ 


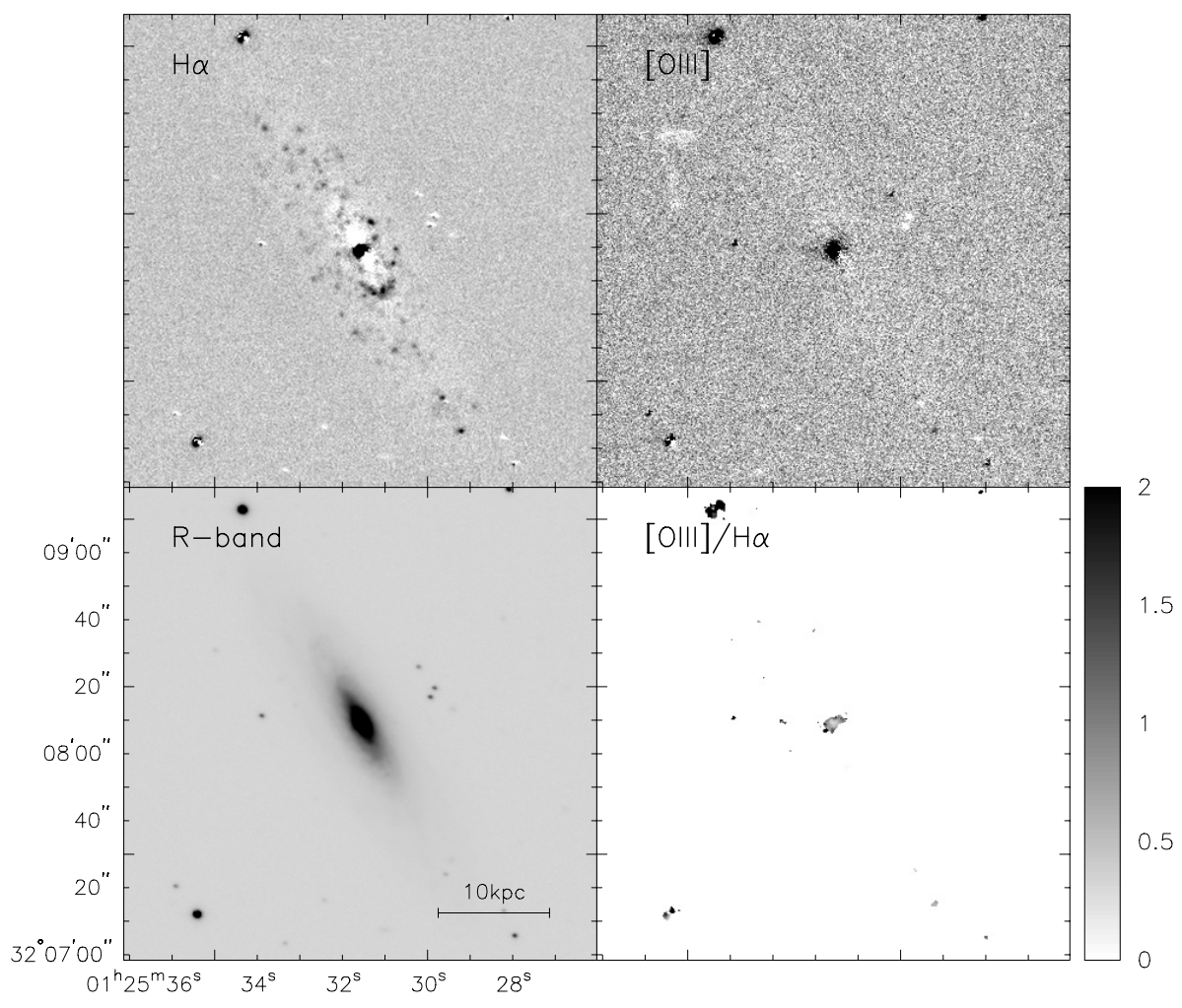

Fig. 5. Broadband, narrowband images and ionization map of Mrk 993. H $\alpha$ (upper left), [O III] (upper right), R-band (lower left), and [O III]/H $\alpha$ (lower right). $(\sim 4.2 \mathrm{kpc})$ around the nucleus. Most of the emission visible in the $[\mathrm{O}$ III $]$ map originates from the nucleus of the galaxy, and none of the widespread emission seen in $\mathrm{H} \alpha$ is seen in [O III]. No extraplanar $\mathrm{H} \alpha$ emission is observed, in agreement with Colbert et al. (1996), and no extraplanar [O III] emission is observed.

\section{2. $M r k 577$}

The disk of Mrk 577 (Fig. 6) is remarkably featureless in the $R$-band, and only the nucleus is visible in the $\mathrm{H} \alpha$ and the [O III] maps. The nucleus in the $\mathrm{H} \alpha$ map is slightly offset in the NW direction compared to the brightest peak in the $R$-band. This may be due to dust extinction in the circumnuclear environment. No planar or extraplanar $\mathrm{H} \alpha$ or [O III] emission is observed for this galaxy. Therefore, only the nucleus is present in the ionization map.

\subsection{UGC 1479}

The $R$-band image of UGC 1479 (Fig. 7) appears relatively featureless apart from the prominent nuclear region. The $\mathrm{H} \alpha$ map clearly shows emission from two spiral arm-like structures, as well as extended emission from the nucleus. The morphology of the latter is reminiscent of a bar joining the base of the two spiral arms. Diffuse $\mathrm{H} \alpha$ emission is observed only within the disk, and extends $32^{\prime \prime}(10.2 \mathrm{kpc})$ to the $\mathrm{N}$ and $25^{\prime \prime}(8.0 \mathrm{kpc})$ to the $\mathrm{S}$ of the nucleus. The [O III] map also reveals emission from the nucleus, and faint diffuse emission from the disk, which seems offset to the E, possibly due to inclination effects. However, this diffuse emission may also be due to residuals from the continuum subtraction. No extraplanar emission is seen in either $\mathrm{H} \alpha$ or [O III]. The black oval region seen in the $R$-band frame is a reflection from a bright star (see Sect. 2.3.2). It appears as a white oval in the $\mathrm{H} \alpha$ image due to the continuum subtraction process.

\subsection{Ark 79}

The $R$-band image of Ark 79 (Fig. 8) shows one nucleus and a structure which may be a faint inner bar. The nucleus is the main contributor to the emission in the $\mathrm{H} \alpha$ and [O III] maps. When shown on a suitable greyscale, the narrowband maps reveal two components to the nucleus (see a zoomed-in version of the nuclear region in Fig. 9). The brightest peak in $\mathrm{H} \alpha$ is located to the $\mathrm{E}$, centered on the broadband peak, and the fainter one is offset to the W. However, the two components have a similar brightness in $[\mathrm{O} \mathrm{III}]$, meaning that the $[\mathrm{O} \mathrm{III}] / \mathrm{H} \alpha$ ionization ratio will be much larger for the offset peak compared to the actual center of the galaxy. The separation between the two components is 2 .'5 $(850 \mathrm{pc})$. This is the first reported detection of a two component nucleus in Ark 79. $\mathrm{H} \alpha$ and [O III] emission is also seen extending into the disk, and resembles the morphology observed by Colbert et al. (1996). No clear extraplanar emission is visible on large scales, also in agreement with Colbert et al. (1996).

\subsection{Mrk 1040}

Our broadband image (see Fig. 10) shows the presence of a companion galaxy $18^{\prime \prime}$ to the $\mathrm{N}$ of the nucleus of Mrk 1040, which may be in the process of merging. This was also previously reported by Colbert et al. (1996). The H $\alpha$ emission from the H II regions in the disk of Mrk 1040 outlines a complex spiral arm structure, with bright $\mathrm{H}$ II regions seen as far out as $119^{\prime \prime}(38.4 \mathrm{kpc})$ to the E of the nucleus, and $86^{\prime \prime}(27.8 \mathrm{kpc})$ to the W. This asymmetry could be explained by interactions with other galaxies such as the northern companion. The nucleus of Mrk 1040 and that of the companion galaxy, as well as one region in the spiral arms $32^{\prime \prime}(10.3 \mathrm{kpc})$ to the $\mathrm{W}$ of the nucleus of Mrk 1040 are strong $\mathrm{H} \alpha$ and [O III] emitters (a closeup view is shown in Fig. 11), and there is a possible a ring-like structure of radius $20^{\prime \prime}(6.5 \mathrm{kpc})$ around the nucleus in $\mathrm{H} \alpha$. The remaining fraction of the $[\mathrm{O}$ III $]$ emission originates from faint diffuse 
emission in the spiral arms. No evidence for extraplanar emission is found in either $\mathrm{H} \alpha$ or [O III]. The ionization map shows mainly the two galactic nuclei and the bright $\mathrm{H}$ II region within the spiral arm.

\subsection{UGC 2936}

Our $R$-band image of UGC 2936 (Fig. 12) reveals the nucleus and $\mathrm{H}$ II regions along spiral arms. These are clearly visible in the $\mathrm{H} \alpha$ map, and are seen scattered throughout the disk out to $68^{\prime \prime}(16.7 \mathrm{kpc})$ to the NE and $58^{\prime \prime}(14.3 \mathrm{kpc})$ to the SW of the nucleus. A few faint clustered H II regions are visible beyond what seems to be the rim of the outer spiral arm. The [O III] map is remarkably featureless, apart from emission from the nucleus.

\subsection{NGC 3079}

Many dusty regions and strong $\mathrm{HII}$ regions are visible in the $R$-band image of NGC 3079 (Fig. 13). Our $\mathrm{H} \alpha$ image shows emission-line regions scattered throughout a warped disk, as well as a superbubble in the nuclear region. Both the superbubble (e.g., Veilleux et al. 1994; Cecil et al. 2001, 2002) and the fueling of active nucleus (e.g., Veilleux et al. 1999b) have been the subject of several previous studies. An enlargement of the inner central region of NGC 3079, tracing the superbubble and the outflow cone, is shown in Fig. 14. The fainter extraplanar gas and large-scale outflows emanating from the disk are shown in an enhanced contrast $\mathrm{H} \alpha$ map (see Fig. 15). It is interesting to note that all the gaseous outflows and extraplanar diffuse emission are only observed to originate within $\sim 70^{\prime \prime}(5.1 \mathrm{kpc})$ on either side of the nucleus. Beyond this radius, the $\mathrm{H} \alpha$ image simply shows bright $\mathrm{H} \alpha$ emission from clumps within the disk, and no extraplanar diffuse emission.

The large-scale outflows extend as far out as $\sim 185^{\prime \prime}$ (for the NE filament) from the disk midplane $(\sim 13.5 \mathrm{kpc})$. The largescale filaments point radially away from regions in the disk of the galaxy rather than away from the nucleus, and seem to trace an X-structure. This was previously observed by Heckman et al. (1990) in $\mathrm{H} \alpha$ (although in a smaller field of view), and by Strickland et al. (2004) in X-ray. Such a morphology is reminiscent of the X-structure observed in NGC 5775 (e.g., Rossa \& Dettmar 2003b) and NGC 4666 (Dahlem et al. 1997), which are starburst galaxies. Our $\mathrm{H} \alpha$ image shows the most extended emission, and is the deepest and largest field of view image of NGC 3079 to date. The SW outflow seems well collimated, and extends out to $\sim 125^{\prime \prime}(9.1 \mathrm{kpc})$. However, we note, that the feature, resembling an outflow to the SE (between the galaxy and the bright star) is a diffraction spike of the stellar image (see also bottom right panel of Fig. 2).

The [O III] image shows emission from compact regions throughout the disk, with the strongest emission occurring to the $\mathrm{N}$ of the nucleus. This region of high [O III] emission seems to be close to the base of one of the disk outflows. The brightest [O III] regions are not associated with the nucleus, which is most likely due to heavy dust extinction close to the nucleus.

\subsection{NGC 3735}

Strong emission from many compact regions in the disk, diffuse emission between these regions, and emission from an extended region around the nucleus are clearly visible in the $\mathrm{H} \alpha$ map (Fig. 16). The [O III] map shows a few compact regions, most of which are off-planar and have counterparts in the $\mathrm{H} \alpha$ map. As shown in high contrast $\mathrm{H} \alpha$ and [O III] maps (Fig. 15), possible extraplanar regions emitting in [O III], but barely visible in $\mathrm{H} \alpha$, are seen $38^{\prime \prime}(6.6 \mathrm{kpc})$ to the NE and $27^{\prime \prime}$ (4.7 kpc) to the $\mathrm{SW}$ of the nucleus. The high inclination of the galaxy makes these regions more likely to be extraplanar than in the galactic disk. The diffuse emission visible in the high-contrast $\mathrm{H} \alpha$ map could, combined with these extraplanar [O III] regions, be signs of minor-axis outflows.

\subsection{NGC 4235}

The $R$-band image of NGC 4235 (Fig. 17) reveals a bright nuclear region, with a faint dust lane visible on either side of the nucleus. All of the $\mathrm{H} \alpha$ and [O III] emission originates from the nucleus. This is in agreement with previous observations (Pogge 1989; Colbert et al. 1996; Rossa \& Dettmar 2003b). Absorption due to the dust lane is visible to the NW of the nucleus in both narrowband images. The absence of $\mathrm{H}$ II regions in the disk and the absence of extraplanar emission in both maps is in agreement with Pogge (1989), although deeper observations by Rossa \& Dettmar (2003b) reveal faint extended emission in $\mathrm{H} \alpha$.

\subsection{NGC 4388}

The $R$-band image of NGC 4388 (Fig. 18) shows a complicated morphology, where the spiral arms are prominently featured in this highly inclined galaxy. Also present are several circumnuclear dusty regions. The narrowband images, however, show a much more intriguing morphology. NGC 4388 is one of the best studied cases of minor axis outflows (e.g., Pogge 1988b, 1989; Veilleux et al. 1999a,b; Yoshida et al. 2002; Veilleux et al. 2003). Our $\mathrm{H} \alpha$ map shows evidence for spiral arms with a number of $\mathrm{H}$ II regions, as well as the nucleus whose morphology is reminiscent of a bar joining the base of the two spiral arms. Also visible in the high-contrast image shown in Figs. 15 and 19 (zoomin) are the outflows extending $57^{\prime \prime}(4.6 \mathrm{kpc})$ to the $\mathrm{N}$ and $31^{\prime \prime}$ $(2.5 \mathrm{kpc})$ to the $\mathrm{S}$ of the midplane of the galaxy. These are only small sections of the large-scale outflows which extend out to distances of $35 \mathrm{kpc}$ from the nucleus (Yoshida et al. 2002). The [O III] map shows strong emission from the nucleus, which extends in the southern direction. Also visible are outflows to the $\mathrm{N}$ and $\mathrm{S}$, corresponding to those seen in $\mathrm{H} \alpha$, and diffuse emission from the western spiral arm. By and large, the nuclear region looks more complex in [O III] than it does in $\mathrm{H} \alpha$.

\subsection{NGC 4565}

The $R$-band image of this highly-inclined Seyfert galaxy (Fig. 20) reveals a very prominent dust lane. Single chimneylike dust structures protrude from the northern plane perpendicular to the disk into the disk-halo interface. The $\mathrm{H} \alpha$ map of NGC 4565 shows clumpy emission line regions and diffuse ionized gas scattered through the disk. No extraplanar $\mathrm{H} \alpha$ emission is observed, in agreement with previous observations performed by Rand et al. (1992). However, there is possible evidence for some faint filaments. The brightest regions to the SE of the nucleus, as well as a few other emission regions in $\mathrm{H} \alpha$ have counterparts in the [O III] image. The disk region that displays the strongest [O III] emission, is shown in Fig. 21. The brightest [O III] source is an off-planar H II region, located to the $\mathrm{N}$ of the disk. The bulge is clearly not perfectly subtracted in the overall 
[O III] image, and this leads to some spuriously low values in the ionization map.

\subsection{NGC 5866}

The $R$-band image of NGC 5866 (Fig. 22) shows a prominent bulge and a dust lane. Unlike other galaxies in our sample, no clumpy $\mathrm{H}$ II regions are seen in $\mathrm{H} \alpha$. The diffuse $\mathrm{H} \alpha$ emission is partially obscured by the prominent dust lane and is asymmetric with respect to the plane of the disk. The [O III] emission is also partially obscured by the dust lane, and does not display any clumpy regions. It is not clear whether the diffuse emission seen on either side of the dust lane in $\mathrm{H} \alpha$ is a real feature and whether this contains evidence for any outflows. More likely its presence is artificial due to difficulties associated with the continuum subtraction. The continuum subtraction proved very delicate for this galaxy and therefore we do not present any flux or ionization ratio measurements.

\subsection{IC 1368}

The $R$-band image of IC 1368 (Fig. 23) shows an X-shaped region of possibly clustered $\mathrm{H}$ II regions. In agreement with Colbert et al. (1996), we find that the $\mathrm{H} \alpha$ image shows a nucleus extended in the minor axis direction. Furthermore, we find evidence for a cone-like structure at the NW tip of the nucleus. In addition to faint diffuse emission from the disk, the $\mathrm{H} \alpha$ emission map shows possible evidence for two spiral arms containing a few bright $\mathrm{H}$ II regions. The [O III] image shows a bright nucleus surrounded by two strong absorption features, as well as diffuse disk emission.

\subsection{UGC 12282}

The $R$-band image of UGC 12282 (Fig. 24) shows some dusty regions in between the modest $\mathrm{H}$ II regions outlining the spiral arms, which are partially visible in this inclined spiral galaxy. The $\mathrm{H} \alpha$ map shows emission from $\mathrm{H}$ II regions scattered in the disk as well as from the nucleus. A ring of enhanced $\mathrm{H} \alpha$ emission with radius $23^{\prime \prime}(7.6 \mathrm{kpc})$ is also visible. The [O III] map does not show much emission outside the nucleus.

\section{Discussion}

\subsection{The ionization maps}

One of the important diagnostics for unraveling the excitation mechanisms in galaxies is the use of emission lines of various excitation levels. In one-dimensional space (i.e. derived from longslit spectra) various measured line ratios (e.g., [N II]/H $\alpha$ or $[\mathrm{O} \mathrm{III}] / \mathrm{H} \beta$ ) can be used to discriminate between AGN, LINER, or H II emission line regions, making use of well known diagnostic diagrams (e.g., Veilleux \& Osterbrock 1987). The advantage of emission line imaging is that it provides two-dimensional coverage. Therefore, we get an excitation map as a function of the position within the galaxy (i.e. for individual regions of interest) and for each covered pixel of the entire galaxy in general. The emission lines used in our study are some of the most prominent ones seen in Seyfert galaxies in the optical regime, $\mathrm{H} \alpha$ and [O III]. We created the [O III] $/ \mathrm{H} \alpha$ ionization maps, shown in the lower right hand panels of Figs. 5 to 24. These maps might have been very useful in the characterization of any newly detected outflows. However, we did not find any new outflows but merely confirm the already well-studied outflows in NGC 3079 and NGC 4388. Therefore, our [O III] $/ \mathrm{H} \alpha$ maps did not provide much new insight in the end, except in the cases which had previously only be studied in $\mathrm{H} \alpha$. Nonetheless, we briefly discuss them here for completeness.

The majority of our ionization maps are restricted to the nuclear and circumnuclear region, as our sensitivity did not reveal much [O III] emission within the disk. However, a few galaxies did reveal off-planar emission and disk emission line regions. In most cases, though we find that it is related to $\mathrm{H}$ II regions and not to diffuse emission originating from outflows. The sole exceptions are the well studied spirals NGC 3079 and NGC 4388. In the case of NGC 3079 the nuclear superbubble is prominently visible in the $[\mathrm{O} \mathrm{III]} / \mathrm{H} \alpha$ ionization map, which has values of about 0.2 to 0.4 . Values of the ratio are higher in the disk, where [O III] emission is quite strong locally, ranging from about 0.3 to 1.0 , with peaks reaching 1.4 or more in some places. By and large the northern part of the disk has the strongest regions (high values of $[\mathrm{O} \mathrm{III]} / \mathrm{H} \alpha \sim 0.7)$, whereas the southern part of the disk regions has considerably lower values ([O III] $/ \mathrm{H} \alpha \sim 0.3$ ).

In the case of NGC 4388 the ionization map shows a "cone" of ionization to the $\mathrm{S}$, in agreement with the measurements of Pogge (1988b). Spectra and diagnostic line ratios presented by Pogge (1988b) showed that the nucleus and the circumnuclear regions can be described by photoionization of the central power-law continuum. In the ionization cone we find $[\mathrm{O} \mathrm{III}] / \mathrm{H} \alpha \sim 1.9$ towards the center of the galaxy. It decreases to 1.4 and increases again to 2.4 further out from the center. The diffuse emission, visible in the two regions at greater extraplanar distances to the $\mathrm{N}$, has much larger ionization values, roughly $2.6 \pm 0.7$ in the upper region, and $4.3 \pm 0.9$ in the lower region, both indicative of shock-ionization.

In the case of Ark 79, the $[\mathrm{O} \mathrm{III}] / \mathrm{H} \alpha$ ratio has a value of about 0.5 for the eastern main nucleus, and about 1.2 for the offset secondary nucleus. The nuclear region of Mrk 1040 has a value of 0.2 to 0.3 , with the most prominent region in the disk of this galaxy having similar ratios of 0.3 to 0.4 . This is lower than the $[\mathrm{O} \mathrm{III}] / \mathrm{H} \alpha$ ratio of the companion galaxy, which is 0.6 .

The emission-line regions seen in the disk of NGC 3735 all have values below 0.7 . We note that the $[\mathrm{O} \mathrm{III]} / \mathrm{H} \alpha$ ratio in the possible extra-planar H II regions cannot be measured due to a too low signal-to-noise ratio. The [O III] $/ \mathrm{H} \alpha$ ratio in the disk of NGC 4565 does not exceed 1.0 in regions where [O III] emission is detected. The majority of the detected regions have $[\mathrm{O}$ III] $/ \mathrm{H} \alpha \leq 0.7$.

The nuclear region of Mrk577 has [O III]/H $\alpha$ values in the range of 0.8 to 1.0. In Mrk993, the value of the ratio varies between 0.2 and 0.9 in the nuclear region, with the innermost region having values closer to 0.6. In UGC 1479, the $[\mathrm{O} \mathrm{III}] / \mathrm{H} \alpha$ ratio varies between 1.0 and 1.4 in the nucleus. Finally, in UGC 2936 the values of the ratio are close to 0.5 in the nuclear region. However, these values should be treated with caution, since the bright nucleus of some of the galaxies is the most sensitive region for the continuum subtraction, and the [O III] or $\mathrm{H} \alpha$ emission seen in the nuclei may be an artifact thereof. The nucleus of NGC 4235 has $[\mathrm{O} \mathrm{III]} / \mathrm{H} \alpha \sim 0.5$, and this is the only region detected in the ratio map.

For NGC 5866 and UGC 12282 the nuclear regions proved unsuitable for the ionization map to permit any meaningful measurement of the ratio, and in IC 1368 poor $\mathrm{S} / \mathrm{N}$ also prevented any confident measurement. 


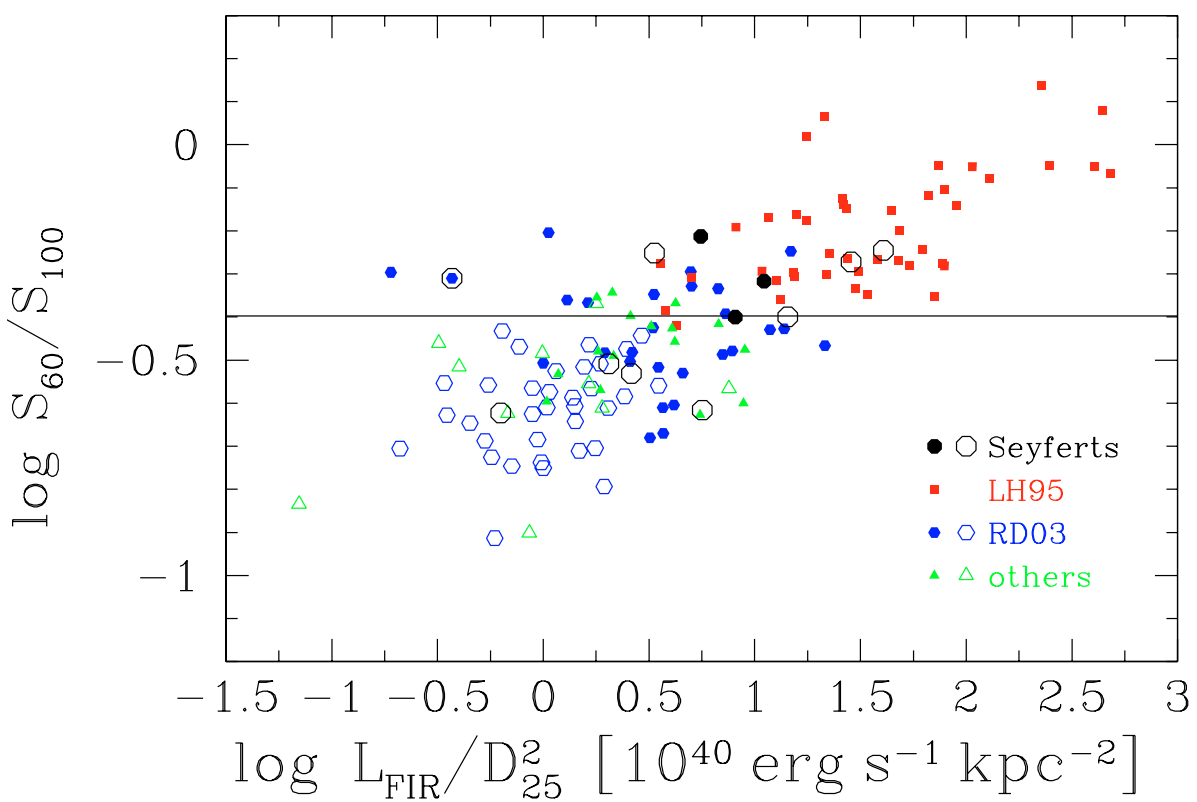

Fig. 25. Diagnostic diagram for extraplanar diffuse ionized gas, originating from disk outflows into the halo of the galaxy, including the Seyfert galaxies studied here (adapted and expanded from Rossa \& Dettmar 2003a). It shows the star formation rate per unit area as a function of the IRAS flux ratio $\left(\log S_{60} / S_{100}\right)$, which is a proxy for the warm dust temperature. Filled symbols indicate galaxies with detected extraplanar diffuse ionized gas, open symbols denote those without. For two of our studied Seyfert galaxies (Mrk 577 and Ark 79) there were no FIR measurements available. Therefore, no data points are plotted for these two objects. The square symbols denote the starburst galaxies studied by Lehnert \& Heckman (1995), the small hexagons denote non-starburst galaxies studied by Rossa \& Dettmar (2003a) and the triangles indicate data points for non-starburst galaxies studied by others (see Rossa \& Dettmar 2003a, for details).

\subsection{Nuclear versus minor axis disk outflows}

The majority of the galaxies studied in this sample show strong nuclear emission, as expected for Seyferts. All but two galaxies (NGC 4565 and NGC 5866) show relatively strong nuclear emission in $\mathrm{H} \alpha$, and most in [O III] too. The two reported exceptions both have a very prominent dust layer, which causes considerable extinction. Four galaxies (Ark 79, NGC 3079, NGC 4388 and IC 1368) have complex circumnuclear emission line regions. In contrast, UGC 1479, Mrk 1040, NGC 3079, NGC 3735 , NGC 4388, NGC 4565 and IC 1368 show off-nuclear (extraplanar) emission line regions. However, not all (e.g., UGC 1479 and Mrk 1040) show diffuse extraplanar emission. A comparison of the relation between star formation rate per unit area with dust temperature is shown in Fig. 25 (see Rossa \& Dettmar 2003a, for more general details). The Seyfert galaxies behave somewhat differently in terms of the location within this diagram than the non-Seyfert galaxies. The majority of the Seyferts (nine versus three) for which FIR data are available, show strong nuclear emission, but have no extended diffuse ionized gas (DIG). This is true even at high FIR luminosity per unit area and high dust temperature. By contrast, in non-Seyfert galaxies this almost uniquely indicates the presence of extraplanar DIG, expelled from the active SF regions in the disk into the halo of these galaxies (e.g., Lehnert \& Heckman 1995; Rossa \& Dettmar 2003a; Miller \& Veilleux 2003). This result is probably not an artifact of differences in sensitivity for detection of DIG between the present study and that of Rossa \& Dettmar (2003a). Both studies used telescopes of similar size with similar observational parameters. Most Seyfert galaxies studied here show no evidence for extended emission line regions in both $\mathrm{H} \alpha$ and [O III]. The activity is solely restricted to the nuclear and circumnuclear environment. Only NGC 3079, NGC 3735 and NGC 4388 show extended DIG emission. NGC 3079 is also known to have a central starburst (Cecil et al. 2002) in addition to its Seyfert nucleus, and that may explain why extended emission is detected. NGC 4388 is a galaxy experiencing ram pressure stripping in the Virgo cluster environment (Kenney et al. 2004), which may be a primary contributor to its very extended emission line regions especially far from the nucleus. So overall it appears that star formation is the primary mechanism producing extended DIG around the spiral galaxies studied by the present study and that of Rossa \& Dettmar (2003a). In general, AGN activity undoubtedly plays some role in driving minor-axis outflows. However, this probably requires higher luminosities than are encountered in our small distance-limited sample.

For Ark 79, which was classified as a type 2 Seyfert galaxy (Osterbrock \& Phillips 1977), we find evidence for a secondary nucleus with a separation of $\approx 2$ '. 5 , which is clearly visible in the [O III] image, but only faintly in the $\mathrm{H} \alpha$ image. The two detected components are aligned roughly along the major axis of the disk. As it is barely detected in $\mathrm{H} \alpha$, the secondary component was undetected in previous studies (e.g., Colbert et al. 1996). The projected distance corresponds roughly to $850 \mathrm{pc}$. This is the only case in our sample which shows a two-component structure of the nuclear region. Apart from this we find at least two more cases with extended circumnuclear regions. NGC 4388 shows a relatively complex circumnuclear region, specifically in [O III], in agreement with previous studies (e.g., Veilleux et al. 1999a; Yoshida et al. 2002). An extended cone-like nuclear outflow region is detected in IC 1368. This is reminiscent of a downsized version of a prominent starburst cone.

\section{Summary}

We have observed a distance limited sample of 14 nearby edgeon Seyfert galaxies with narrowband imaging in $\mathrm{H} \alpha$ and [O III] to study the role of the active nucleus and SF regions within the disk for the IGM enrichment. Because of the distancelimited nature of the sample, it is restricted to relatively lowluminosity Seyfert galaxies. The median 3- $\sigma$ sensitivities for detection of high-latitude extended emission in the sample galaxies are $3.6 \times 10^{-17} \mathrm{erg} \mathrm{s}^{-1} \mathrm{~cm}^{-2} \operatorname{arcsec}^{-2}$ for the $\mathrm{H} \alpha$ images and $6.9 \times 10^{-17} \mathrm{erg} \mathrm{s}^{-1} \mathrm{~cm}^{-2} \operatorname{arcsec}^{-2}$ for the [O III] images. The studied galaxies show a variety of emission line features. The majority, however, reveal only emission in the nuclear and circumnuclear environment at the sensitivity of our observations. Extraplanar emission from strong SF regions in the disk is only observed in three cases (NGC 3079, NGC 3735 and NGC 4388), with filaments reaching distances of up to $13.5 \mathrm{kpc}$ above/below the disk in NGC 3079. Single very faint filaments also seem to originate from strong SF disk regions in NGC 4565, although only to much lower galactic latitudes. In Ark 79 we detect a 
secondary nuclear component, most prominently seen in the [O III] image. The two components are separated by $850 \mathrm{pc}$.

Whereas the $\mathrm{H} \alpha$ emission often shows a variety of discrete features (filaments, emission line cones, $\mathrm{H}$ II regions), the [O III] emission distribution is, with very few exceptions, mainly restricted to the nucleus and in a few cases to the circumnuclear environment. This is probably due to our limited sensitivity, combined with the fact that extended gas, except in very exceptional circumstances, is expected to be relatively lowexcitation (based on ionization parameter arguments considering low-density gas very far from an AGN). In total we detected large scale minor axis outflows only in three of the studied 14 galaxies. The corresponding detection rate of $21 \%$ is similar to the detection rate found by Colbert et al. (1996), which is $27 \%$. The study of Veilleux et al. (2003) had higher sensitivity than both of these studies. However, the results of this latter paper cannot be used for statistical purposes because their sample was pre-selected to contain objects with known ionization cones and/or galactic winds on a roughly kiloparsec scale.

Overall, our results show that extraplanar emission of similar brightness and extent as in the previously known cases of NGC 3079 and NGC 4388 is not common in Seyfert galaxies of otherwise similar properties. In both these galaxies there are external effects, such as a starburst wind or ram pressure stripping, that might effect the extended emission. Comparison with the results of Rossa \& Dettmar (2003a) shows that for nearby edge-on spiral galaxies star formation may be a more powerful mechanism for producing DIG than AGN activity. In general, AGN activity undoubtedly plays some role in driving minor-axis outflows. However, this probably requires higher AGN luminosities than are enountered in our small distance-limited sample. Future studies with larger telescopes and better sensitivity will be helpful to shed more light onto the role of minor axis disk outflows in edge-on Seyfert galaxies.

Acknowledgements. D.J.B. and J.R. acknowledge financial support for this project by the DFG through grants BO 1642/2-1 and BO 1642/3-1. T.R. wishes to thank the Space Telescope Science Institute for repeated hospitality in hosting him as both a summer student and a visitor. We thank the anonymous referee for suggestions that helped improve our paper. This research has made use of the NASA/IPAC Extragalactic Database (NED) which is operated by the Jet Propulsion Laboratory, California Institute of Technology, under contract with the National Aeronautics and Space Administration.

\section{References}

Cayatte, V., Kotanyi, C., Balkowski, C., \& van Gorkom, J. H. 1994, AJ, 107, 1003

Cecil, G., Bland-Hawthorn, J., Veilleux, S., \& Filippenko, A. V. 2001, ApJ, 555, 338

Cecil, G., Bland-Hawthorn, J., \& Veilleux, S. 2002, ApJ, 576, 745

Colbert, E. J. M., Baum, S. A., Gallimore, J. F., et al. 1996, ApJS, 105, 75

Dahlem, M., Petr, M. G., Lehnert, M. D., Heckman, T. M., \& Ehle, M. 1997, A\&A, 320, 731

Heckman, T. M., Armus, L., \& Miley, G. K. 1990, ApJS, 74, 833

Kenney, J. D. P., \& Koopmann, R. A. 1999, AJ, 117, 181

Kenney, J. D. P., van Gorkom, J. H., \& Vollmer, B. 2004, AJ, 127, 3361

Landolt, A. U. 1992, AJ, 104, 340

Lehnert, M. D., \& Heckman, T. M. 1995, ApJS, 97, 89

Lehnert, M. D., \& Heckman, T. M. 1996a, ApJ, 462, 651

Lehnert, M. D., \& Heckman, T. M. 1996b, ApJ, 472, 546

Miller, S. T., \& Veilleux, S. 2003, ApJS, 148, 383

Osterbrock, D. E., \& Phillips, M. M. 1977, PASP, 89, 251

Pogge, R. W. 1988a, ApJ, 328, 519

Pogge, R. W. 1988b, ApJ, 332, 702

Pogge, R. W. 1989, ApJ, 345, 730

Rand, R. J. 1996, ApJ, 462, 712

Rand, R. J., Kulkarni, S. R., \& Hester, J. J. 1992, ApJ, 396, 97

Reif, K., Bagschik K., de Boer K. S., et al. 1999, SPIE, 3649, 109

Reynolds, R. J. 1992, ApJ, 392, L35

Rossa, J., \& Dettmar, R.-J. 2000, A\&A, 359, 433

Rossa, J., \& Dettmar, R.-J. 2003a, A\&A, 406, 493

Rossa, J., \& Dettmar, R.-J. 2003b, A\&A, 406, 505

Strickland, D. K., Heckman, T. M., Colbert, E. J. M., Hoopes, C. G., \& Weaver, K. A. 2004, ApJS, 151, 193

Veilleux, S., \& Osterbrock, D. E. 1987, ApJS, 63, 295

Veilleux, S., \& Rupke, D. S. 2002, ApJ, 565, L63

Veilleux, S., Cecil, G., Bland-Hawthorn, J., et al. 1994, ApJ, 433, 48

Veilleux, S., Cecil, G., \& Bland-Hawthorn, J. 1995, ApJ, 445, 152

Veilleux, S., Bland-Hawthorn, J., Cecil, G., Tully, R. B., \& Miller, S. T. 1999a, ApJ, 520, 111

Veilleux, S., Bland-Hawthorn, J., \& Cecil, G. 1999b, AJ, 118, 2108

Veilleux, S., Shopbell, P. L., Rupke, D. S., Bland-Hawthorn, J., \& Cecil, G. 2003, AJ, 126, 2185

Veilleux, S., Cecil, G., \& Bland-Hawthorn, J. 2005, ARA\&A, 43, 769

Véron-Cetty, M. P., \& Véron, P. 2001, A\&A, 374, 92

Walker, I. R., Mihos, J. C., \& Hernquist, L. 1996, ApJ, 460, 121

Yoshida, M., Yagi, M., Okamura, S., et al. 2002, ApJ, 567, 118

Yoshida, M., Ohyama, Y., Iye, M., et al. 2004, AJ, 127, 90 
T. P. Robitaille et al.: Gaseous outflows in edge-on Seyfert galaxies, Online Material $p 1$

\section{Online Material}


Table 2. Journal of observations.

\begin{tabular}{|c|c|c|c|c|c|c|c|c|}
\hline Galaxy & $\begin{array}{c}\text { Date } \\
\text { [mm-dd-yyyy }] \\
\text { (2) }\end{array}$ & $\begin{array}{c}\lambda(\mathrm{H} \alpha) \\
{[\AA]} \\
(3)\end{array}$ & $\begin{array}{c}\Delta \lambda(\mathrm{H} \alpha) \\
{[\AA]} \\
(4)\end{array}$ & $\begin{array}{c}t_{\exp }(\mathrm{H} \alpha \& \mathrm{R}) \\
{[\mathrm{s}]} \\
(5)\end{array}$ & $\begin{array}{c}\lambda([\mathrm{O} \text { III }]) \\
{[\AA]} \\
(6)\end{array}$ & $\begin{array}{c}\Delta \lambda([\mathrm{O} \mathrm{III}]) \\
{[\AA]} \\
(7)\end{array}$ & $\begin{array}{c}t_{\exp }([\mathrm{O} \mathrm{III}] \& \mathrm{~B}) \\
{[\mathrm{sec}]} \\
(8)\end{array}$ & $\begin{array}{l}\text { Seeing } \\
\text { [arcsec] } \\
\text { (9) }\end{array}$ \\
\hline \multirow[t]{2}{*}{ Mrk 993} & $10 / 28 / 2002$ & 6667 & 76 & $6 \times 1200$ & 5066 & 104 & $6 \times 1200$ & \\
\hline & & & $\ldots$ & $3 \times 300+1 \times 600$ & & & $3 \times 300+1 \times 600$ & 1.1 \\
\hline \multirow[t]{2}{*}{ Mrk 577} & $10 / 29 / 2002$ & 6667 & 76 & $6 \times 1200$ & 5066 & 104 & $6 \times 1200$ & \\
\hline & & $\ldots$ & $\ldots$ & $3 \times 600$ & $\ldots$ & & $3 \times 600$ & 0.9 \\
\hline \multirow[t]{2}{*}{ UGC 1479} & $10 / 30 / 2002$ & 6667 & 76 & $3 \times 2400$ & 5066 & 104 & $3 \times 2400$ & \\
\hline & & & & $3 \times 600$ & & & $3 \times 600$ & 1.0 \\
\hline \multirow[t]{2}{*}{ Ark 79} & $10 / 28,30 / 2002$ & 6667 & 76 & $6 \times 1200$ & 5066 & 104 & $6 \times 1200$ & \\
\hline & & & $\ldots$ & $2 \times 600$ & $\ldots$ & $\ldots$ & $2 \times 600$ & 1.3 \\
\hline \multirow[t]{2}{*}{ Mrk 1040} & $10 / 30 / 2002$ & 6667 & 76 & $1 \times 1200+2 \times 2400$ & 5066 & 104 & $1 \times 1200+2 \times 2400$ & \\
\hline & & $\ldots$ & $\ldots$ & $2 \times 600$ & $\ldots$ & $\ldots$ & $2 \times 600$ & 1.3 \\
\hline \multirow[t]{2}{*}{ UGC 2936} & $10 / 29 / 2002$ & 6667 & 76 & $6 \times 1200$ & 5066 & 104 & $6 \times 1200$ & \\
\hline & & & $\cdots$ & $2 \times 600$ & 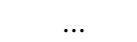 & $\ldots$ & $2 \times 600$ & 0.9 \\
\hline \multirow[t]{2}{*}{ NGC 3079} & $04 / 05 / 2003$ & 6575 & 93 & $4 \times 1800$ & 5007 & 87 & $4 \times 1800$ & \\
\hline & & & & $2 \times 600$ & & & $2 \times 600$ & 1.6 \\
\hline \multirow[t]{2}{*}{ NGC 3735} & $04 / 06 / 2003$ & 6575 & 93 & $3 \times 2400$ & 5007 & 87 & $3 \times 2400$ & \\
\hline & & & & $2 \times 600$ & & & $2 \times 600$ & 1.3 \\
\hline \multirow[t]{2}{*}{ NGC 4235} & $04 / 05,06 / 2003$ & 6575 & 93 & $2 \times 1200+2 \times 2400$ & 5007 & 87 & $2 \times 1200+2 \times 2400$ & \\
\hline & & $\ldots$ & $\cdots$ & $3 \times 600$ & $\ldots$ & $\cdots$ & $3 \times 600$ & 1.5 \\
\hline \multirow[t]{2}{*}{ NGC 4388} & $04 / 05 / 2003$ & 6575 & 93 & $1 \times 2400$ & 5007 & 87 & $1 \times 2400$ & \\
\hline & & & & $1 \times 600$ & & & $1 \times 600$ & 1.4 \\
\hline \multirow[t]{2}{*}{ NGC 4565} & $04 / 05 / 2003$ & 6575 & 93 & $4 \times 1800$ & 5007 & 87 & $4 \times 1800$ & \\
\hline & & & $\ldots$ & $2 \times 600$ & 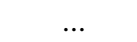 & & $2 \times 600$ & 1.6 \\
\hline \multirow[t]{2}{*}{ NGC 5866} & $04 / 05,06 / 2003$ & 6575 & 93 & $1 \times 1800+2 \times 2400$ & 5007 & 87 & $1 \times 1800+2 \times 2400$ & \\
\hline & & & $\cdots$ & $2 \times 600$ & $\ldots$ & $\ldots$ & $2 \times 600$ & 1.3 \\
\hline \multirow[t]{2}{*}{ IC 1368} & $10 / 28,29 / 2002$ & 6667 & 76 & $6 \times 1200$ & 5066 & 104 & $6 \times 1200$ & \\
\hline & & & $\ldots$ & $3 \times 600$ & $\ldots$ & $\ldots$ & $3 \times 600$ & 1.1 \\
\hline \multirow[t]{2}{*}{ UGC 12282} & $10 / 30 / 2002$ & 6667 & 76 & $3 \times 2400$ & 5066 & 104 & $3 \times 2400$ & \\
\hline & & $\ldots$ & $\ldots$ & $2 \times 600$ & $\ldots$ & $\ldots$ & $2 \times 600$ & 0.9 \\
\hline
\end{tabular}

Notes: column (1) lists the galaxy name. Column (2) lists the date of observation. Columns (3) and (4) give the central wavelength of the H $\alpha$ filter and the FWHM, respectively. Column (5) gives the total $\mathrm{H} \alpha$ exposure time on the top line for each object and the total $R$-band exposure time on the second line. Columns (6) and (7) list the central wavelength of the [O III] filter and the FWHM, respectively. Note, that for the $B$-and $R$-band we do not list a central wavelength and FWHM, as the dichroic filters were used (see Sect. 2.2 and Fig. 1). Column (8) gives the total [O III] exposure time on the top line for each object and the total $B$-band exposure time on the second line. Column (9) lists the average seeing for each galaxy. This was found by measuring the PSF FWHM on the final broadband and narrowband images, and computing the mean of the four values obtained. 
T. P. Robitaille et al.: Gaseous outflows in edge-on Seyfert galaxies, Online Material $p 3$

Table 5. Sensitivity limits for the $\mathrm{H} \alpha$ and [O III] emission-line maps.

\begin{tabular}{|c|c|c|c|c|c|c|}
\hline \multirow{3}{*}{$\begin{array}{l}\text { Galaxy } \\
\text { (1) }\end{array}$} & \multicolumn{2}{|c|}{ Predicted $1-\sigma$ noise } & \multicolumn{2}{|c|}{ Measured 1- $\sigma$ noise } & \multicolumn{2}{|c|}{ Predicted 3- $\sigma$ noise } \\
\hline & \multicolumn{2}{|c|}{$\left[\mathrm{erg} \mathrm{s}^{-1} \mathrm{~cm}^{-2} \operatorname{arcsec}^{-2}\right]$} & \multicolumn{2}{|c|}{$\begin{array}{l}\mathrm{H} \alpha \\
{\left[\mathrm{erg} \mathrm{s}^{-1} \mathrm{~cm}^{-2} \operatorname{arcsec}^{-2}\right]}\end{array}$} & \multicolumn{2}{|c|}{$\begin{array}{cc}\mathrm{H} \alpha & {[\mathrm{O} \mathrm{III]}]} \\
{\left[\mathrm{erg} \mathrm{s}^{-1} \mathrm{~cm}^{-2} \operatorname{arcsec}^{-2}\right]}\end{array}$} \\
\hline & (2) & (3) & (4) & (5) & (6) & (7) \\
\hline Mrk 993 & $1.14 \times 10^{-17}$ & $2.56 \times 10^{-17}$ & $1.03 \times 10^{-17}$ & $2.97 \times 10^{-17}$ & $3.41 \times 10^{-17}$ & $7.69 \times 10^{-17}$ \\
\hline Mrk 577 & $1.03 \times 10^{-17}$ & $2.20 \times 10^{-17}$ & $8.62 \times 10^{-18}$ & $2.72 \times 10^{-17}$ & $3.10 \times 10^{-17}$ & $6.59 \times 10^{-17}$ \\
\hline UGC 1479 & $8.61 \times 10^{-18}$ & $1.73 \times 10^{-17}$ & $8.21 \times 10^{-18}$ & $2.07 \times 10^{-17}$ & $2.58 \times 10^{-17}$ & $5.18 \times 10^{-17}$ \\
\hline Ark 79 & $1.25 \times 10^{-17}$ & $2.71 \times 10^{-17}$ & $1.10 \times 10^{-17}$ & $2.98 \times 10^{-17}$ & $3.74 \times 10^{-17}$ & $8.12 \times 10^{-17}$ \\
\hline Mrk 1040 & $1.20 \times 10^{-17}$ & $2.48 \times 10^{-17}$ & $1.17 \times 10^{-17}$ & $2.68 \times 10^{-17}$ & $3.61 \times 10^{-17}$ & $7.44 \times 10^{-17}$ \\
\hline UGC 2936 & $1.28 \times 10^{-17}$ & $2.71 \times 10^{-17}$ & $1.19 \times 10^{-17}$ & $3.05 \times 10^{-17}$ & $3.85 \times 10^{-17}$ & $8.13 \times 10^{-17}$ \\
\hline NGC 3079 & $1.20 \times 10^{-17}$ & $1.72 \times 10^{-17}$ & $1.22 \times 10^{-17}$ & $2.12 \times 10^{-17}$ & $3.59 \times 10^{-17}$ & $5.16 \times 10^{-17}$ \\
\hline NGC 3735 & $2.44 \times 10^{-17}$ & $2.97 \times 10^{-17}$ & $2.19 \times 10^{-17}$ & $3.29 \times 10^{-17}$ & $7.32 \times 10^{-17}$ & $8.92 \times 10^{-17}$ \\
\hline NGC 4235 & $1.51 \times 10^{-17}$ & $2.39 \times 10^{-17}$ & $1.21 \times 10^{-17}$ & $2.66 \times 10^{-17}$ & $4.54 \times 10^{-17}$ & $7.17 \times 10^{-17}$ \\
\hline NGC 4388 & $3.10 \times 10^{-17}$ & $4.43 \times 10^{-17}$ & $2.32 \times 10^{-17}$ & $4.09 \times 10^{-17}$ & $9.31 \times 10^{-17}$ & $1.33 \times 10^{-16}$ \\
\hline NGC 4565 & $1.13 \times 10^{-17}$ & $1.74 \times 10^{-17}$ & $9.94 \times 10^{-18}$ & $2.21 \times 10^{-17}$ & $3.39 \times 10^{-17}$ & $5.21 \times 10^{-17}$ \\
\hline NGC 5866 & $1.23 \times 10^{-17}$ & $1.92 \times 10^{-17}$ & $1.06 \times 10^{-17}$ & $2.19 \times 10^{-17}$ & $3.68 \times 10^{-17}$ & $5.76 \times 10^{-17}$ \\
\hline IC 1368 & $9.09 \times 10^{-18}$ & $1.81 \times 10^{-17}$ & $8.44 \times 10^{-18}$ & $2.38 \times 10^{-17}$ & $2.73 \times 10^{-17}$ & $5.42 \times 10^{-17}$ \\
\hline UGC 12282 & $8.56 \times 10^{-18}$ & $1.59 \times 10^{-17}$ & $7.74 \times 10^{-18}$ & $1.96 \times 10^{-17}$ & $2.57 \times 10^{-17}$ & $4.78 \times 10^{-17}$ \\
\hline
\end{tabular}

Notes: column (1) lists the galaxy name. Columns (2) and (3) list the 1- $\sigma$ noise levels found from computing the total noise arising from the Poisson noise of the sky background and the readout noise of the CCD chips. Columns (4) and (5) list the 1- $\sigma$ noise levels measured in the emission-line maps. Columns (6) and (7) list the predicted 3- $\sigma$ noise level (i.e. Cols. (2) and (3) multiplied by 3).

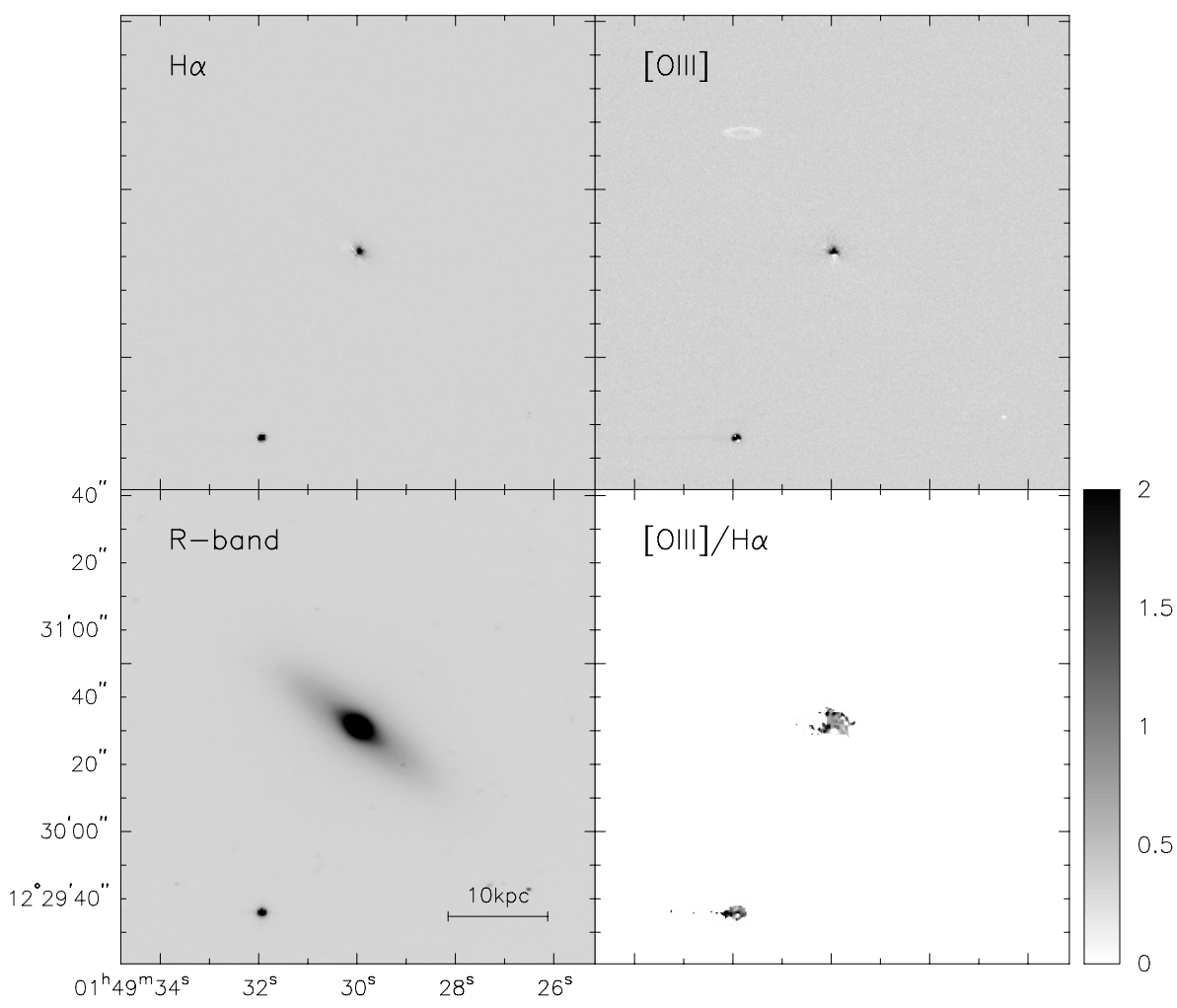

Fig. 6. Broadband, narrowband images and ionization map of Mrk 577. H $\alpha$ (upper left), [O III] (upper right), R-band (lower left), and $[\mathrm{O} \mathrm{III}] / \mathrm{H} \alpha$ (lower right). 
T. P. Robitaille et al.: Gaseous outflows in edge-on Seyfert galaxies, Online Material p 4
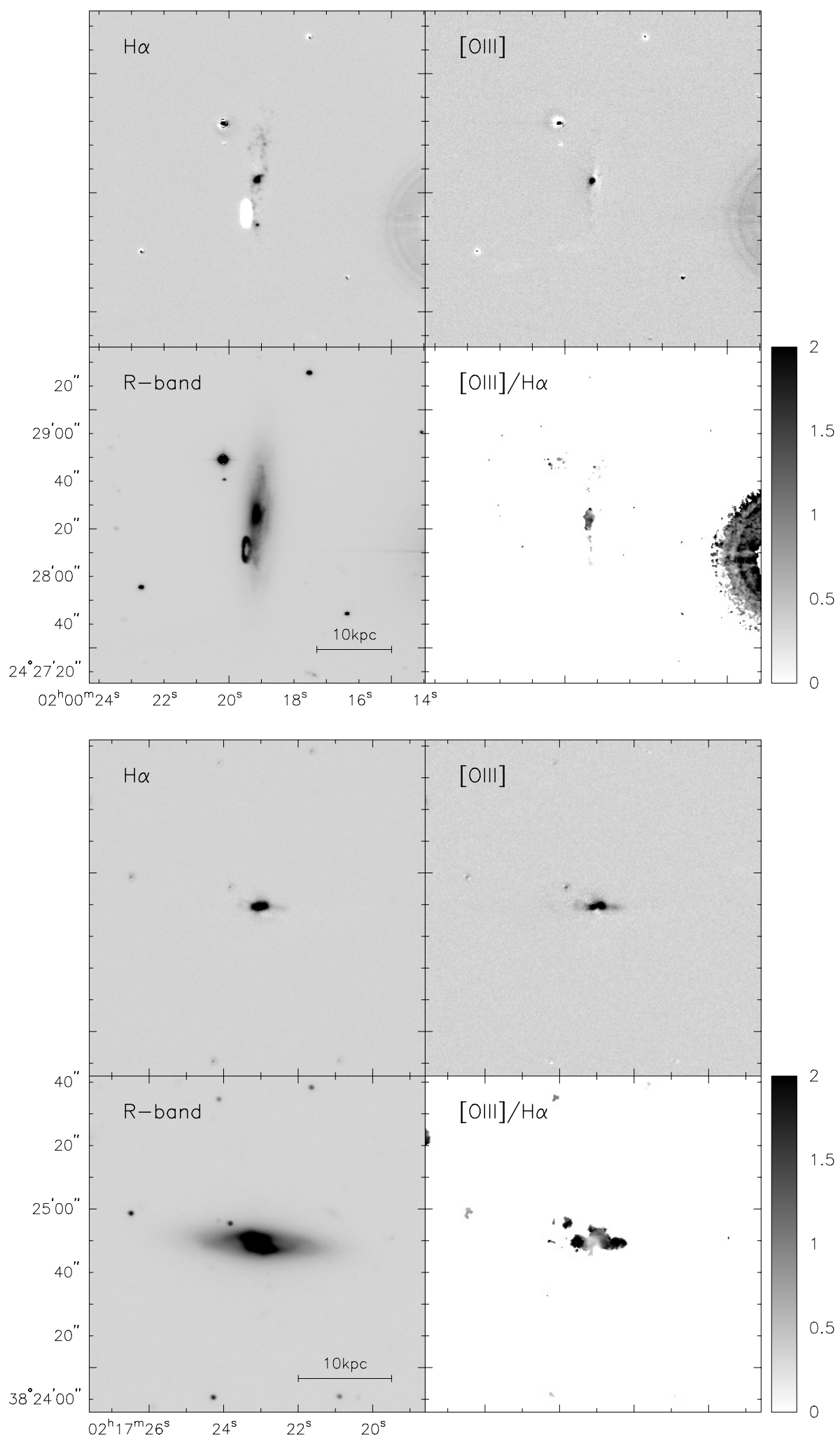

Fig. 7. Broadband, narrowband images and ionization map of UGC 1479. H $\alpha$ (upper left), [O III] (upper right), $R$-band (lower left), and $[\mathrm{O}$ III $] / \mathrm{H} \alpha$ (lower right). The oval white region in the $\mathrm{H} \alpha$ map and the corresponding feature in the $R$-band image close to the southern part of the disk is a ghost of a bright star just off to the right of the displayed field of view (see Sect. 2.3.2).

Fig. 8. Broadband, narrowband images and ionization map of Ark 79. H $\alpha$ (upper left), [O III] (upper right), $R$-band (lower left), and $[\mathrm{O} \mathrm{III}] / \mathrm{H} \alpha$ (lower right). 
T. P. Robitaille et al.: Gaseous outflows in edge-on Seyfert galaxies, Online Material p 5
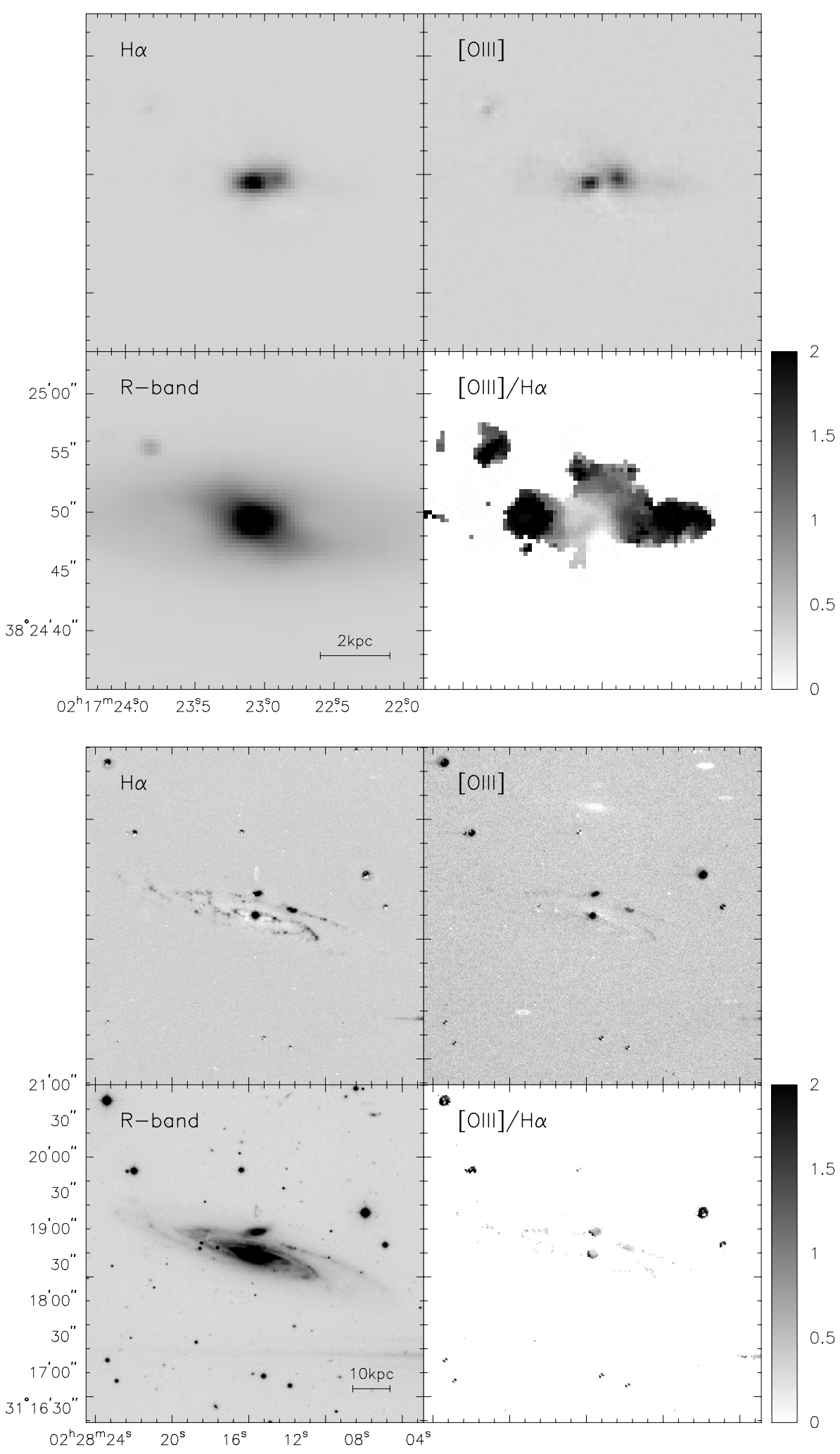

Fig. 9. A blow-up version of Fig. 8, showing the nuclear region of Ark 79. The $\mathrm{H} \alpha$ and [O III] images are also shown in a slightly different greyscale stretch, to highlight the two components more clearly.
Fig. 10. Broadband, narrowband images and ionization map of Mrk 1040. H $\alpha$ (upper left), [O III] (upper right), R-band (lower left), and $[\mathrm{O} \mathrm{III}] / \mathrm{H} \alpha$ (lower right). 
T. P. Robitaille et al.: Gaseous outflows in edge-on Seyfert galaxies, Online Material $p 6$
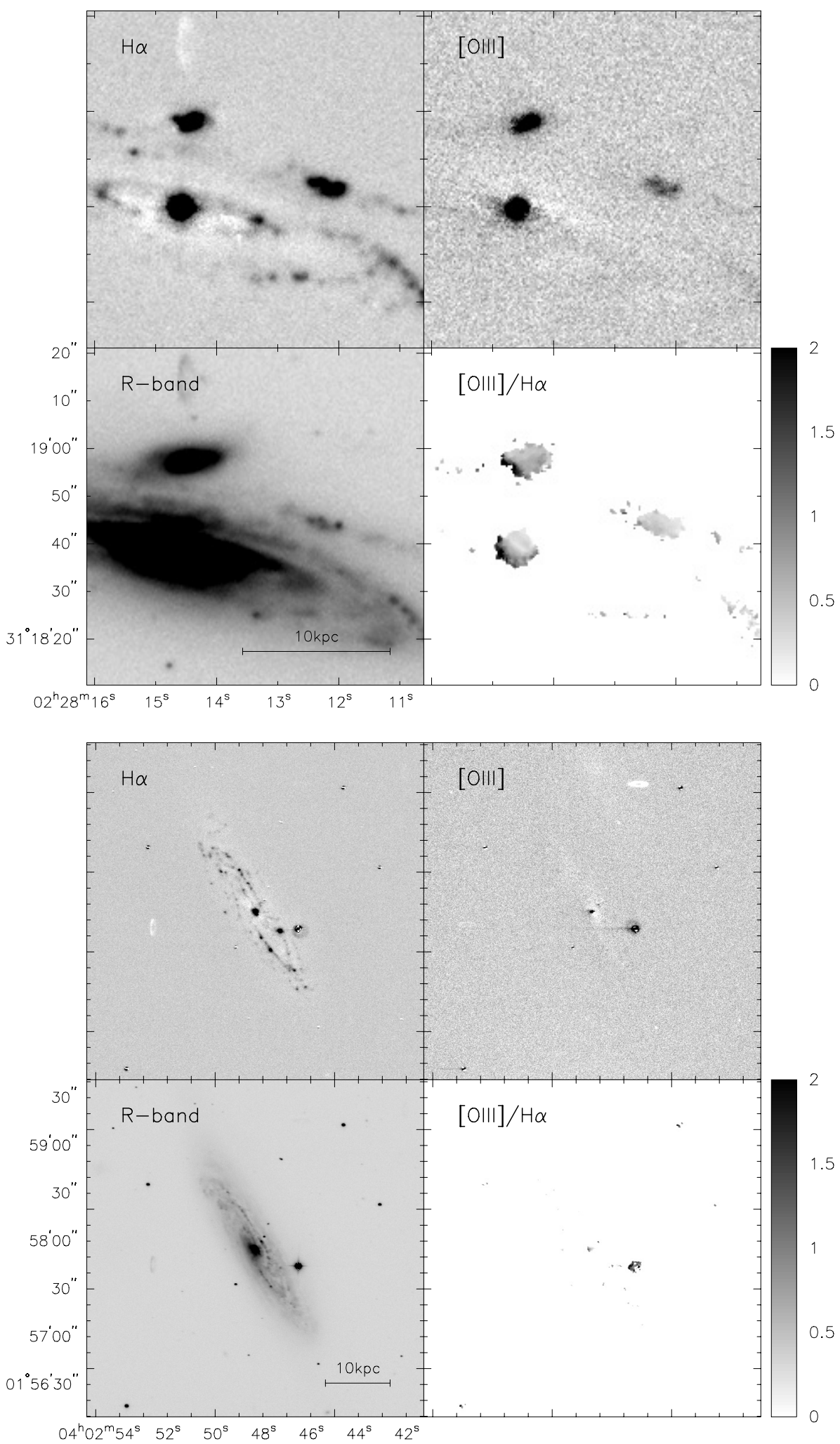

Fig. 11. A zoom-in of Fig. 10, highlighting the central parts of Mrk 1040.

Fig. 12. Broadband, narrowband images and ionization map of UGC 2936. H $\alpha$ (upper left), [O III] (upper right), R-band (lower left), and $[\mathrm{O} \mathrm{III}] / \mathrm{H} \alpha$ (lower right). 
T. P. Robitaille et al.: Gaseous outflows in edge-on Seyfert galaxies, Online Material $p 7$
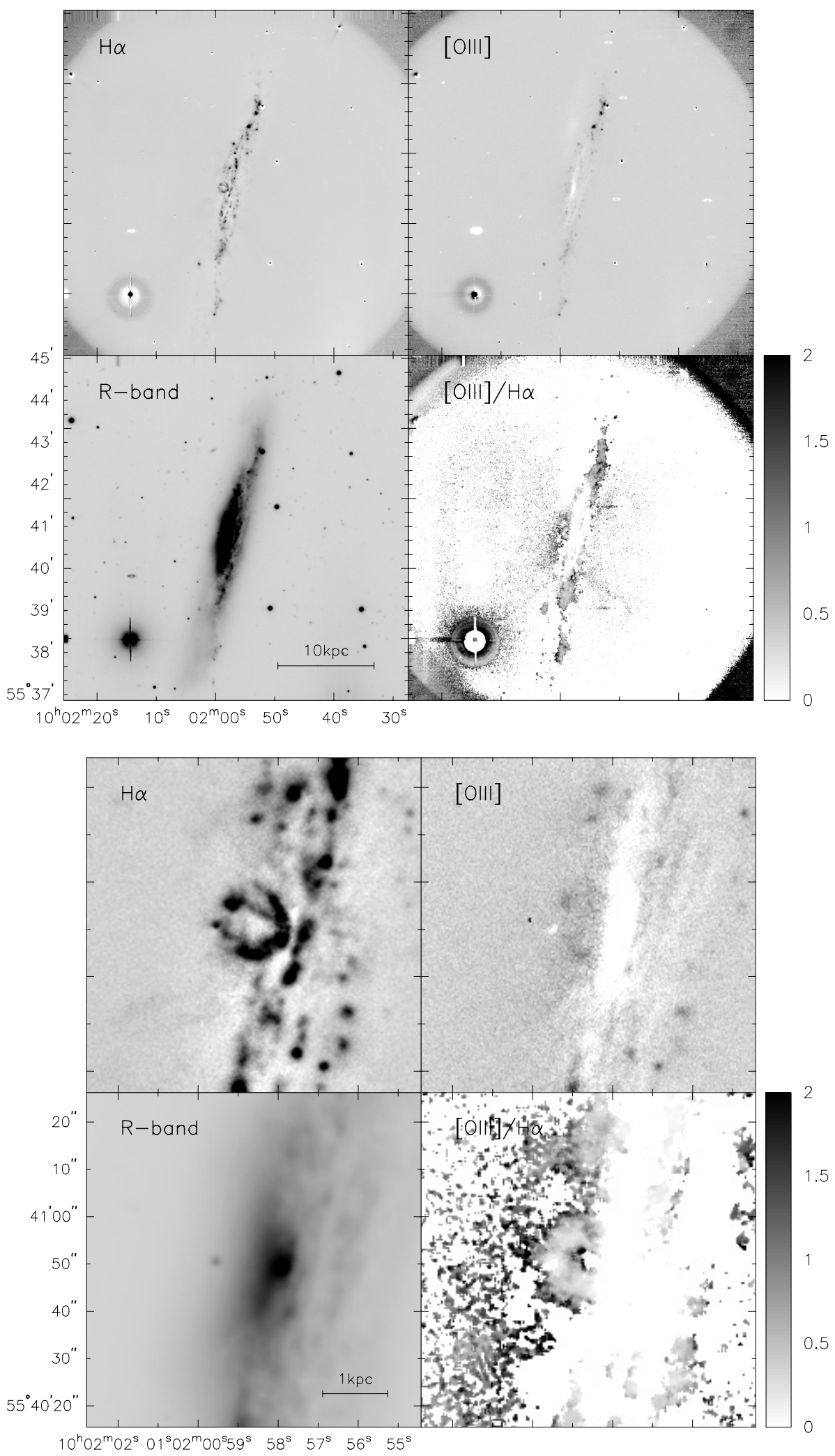

Fig. 13. Broadband, narrowband images and ionization map of NGC 3079. H $\alpha$ (upper left), [O III] (upper right), $R$-band (lower left), and [O III]/H $\alpha$ (lower right).
Fig. 14. A zoom-in of Fig. 13, showing the central part (including the superbubble) of NGC 3079. 
T. P. Robitaille et al.: Gaseous outflows in edge-on Seyfert galaxies, Online Material p 8
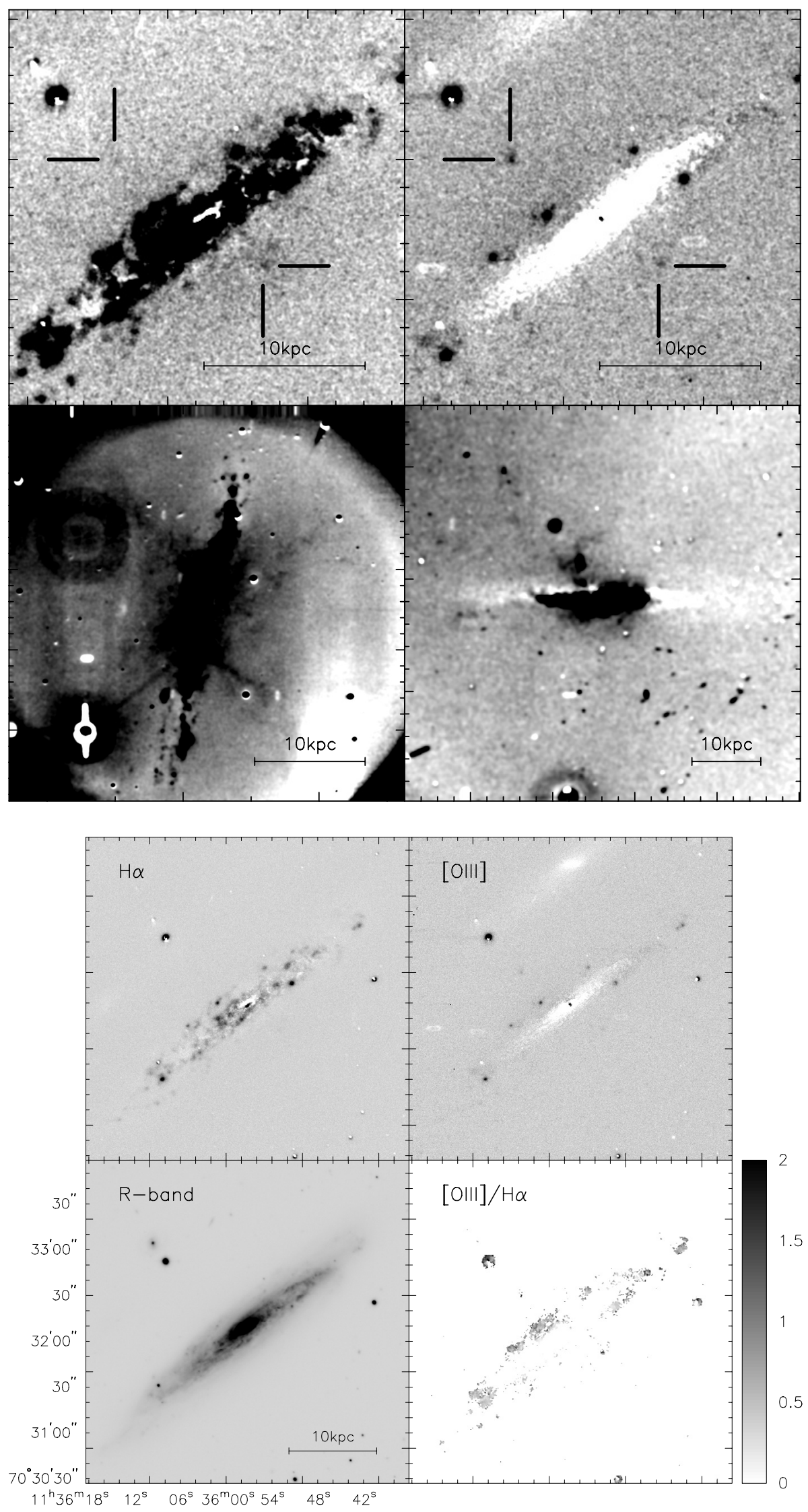

Fig. 15. High contrast images of NGC 3079 in $\mathrm{H} \alpha$ (lower left), NGC 3735 in $\mathrm{H} \alpha$ (upper left), NGC 3735 in [O III] (upper right) and NGC 4388 in $\mathrm{H} \alpha$ (lower right). Note, that there are some remaining cosmic-ray events (the dark spots mostly to the $\mathrm{S}$ of the galaxy) in the $\mathrm{H} \alpha$ image of NGC 4388, since only one single image was obtained for this galaxy.

Fig. 16. Broadband, narrowband images and ionization map of NGC 3735. H $\alpha$ (upper left), [O III] (upper right), $R$-band (lower left), and $[\mathrm{O} \mathrm{III}] / \mathrm{H} \alpha$ (lower right). 
T. P. Robitaille et al.: Gaseous outflows in edge-on Seyfert galaxies, Online Material $p 9$
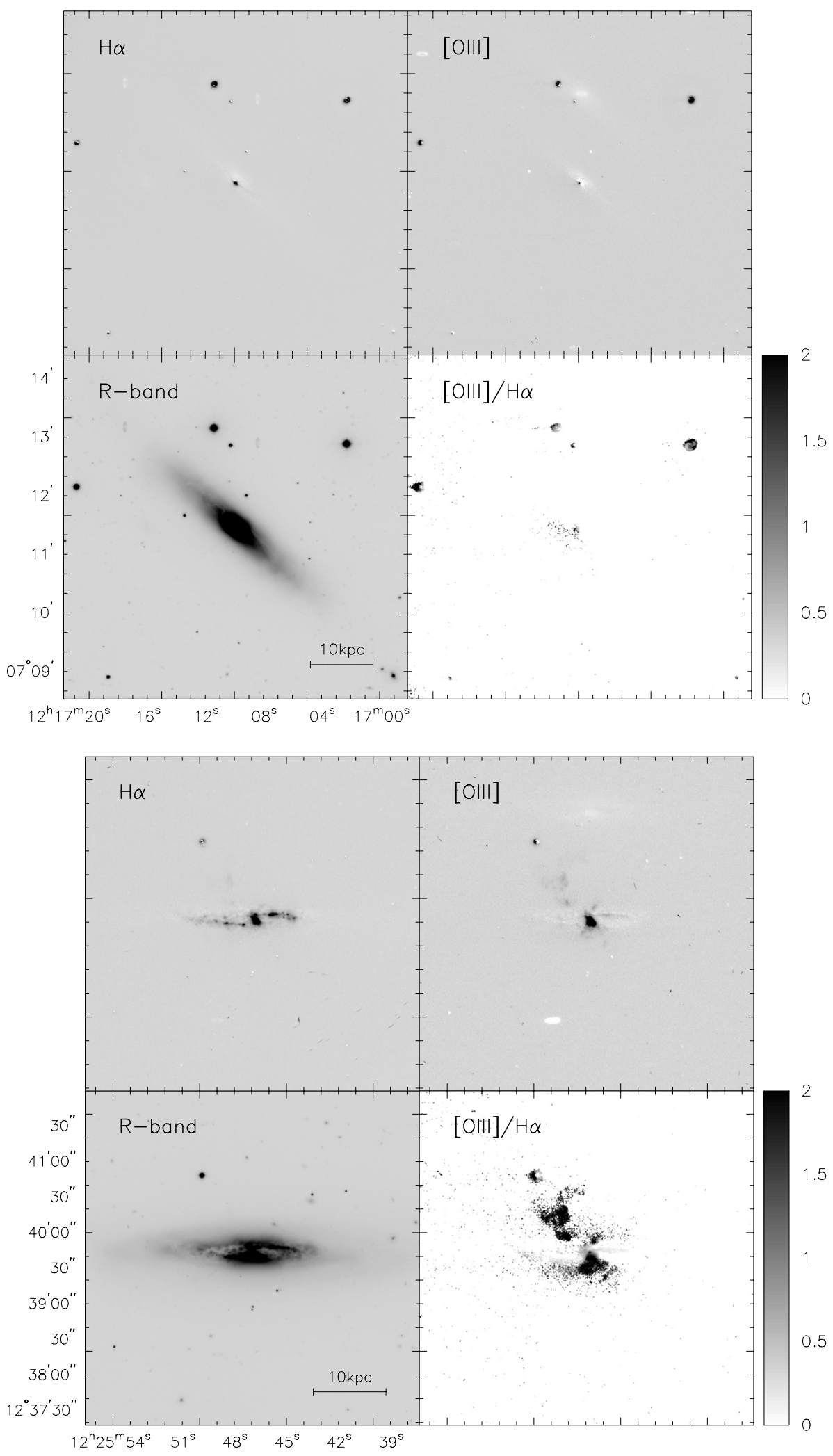

Fig. 17. Broadband, narrowband images and ionization map of NGC 4235. H $\alpha$ (upper left), [O III] (upper right), $R$-band (lower left), and $[\mathrm{O} \mathrm{III}] / \mathrm{H} \alpha$ (lower right).

Fig. 18. Broadband, narrowband images and ionization map of NGC 4388. H $\alpha$ (upper left), [O III] (upper right), R-band (lower left), and [O III] $/ \mathrm{H} \alpha$ (lower right). 
T. P. Robitaille et al.: Gaseous outflows in edge-on Seyfert galaxies, Online Material $p 10$
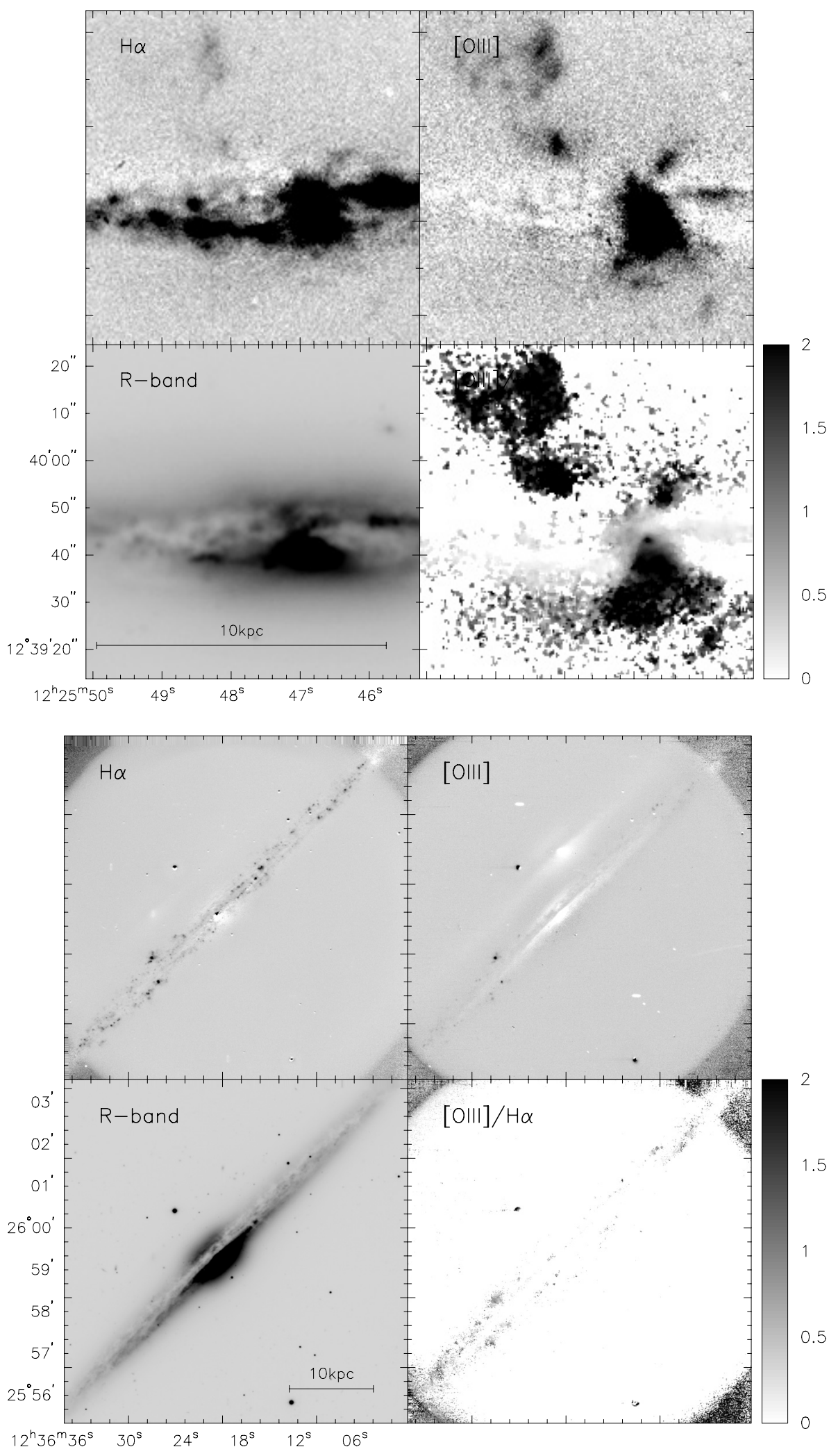

Fig. 19. Zoom-in version of NGC 4388 in different contrast as compared to Fig. 18.

Fig. 20. Broadband, narrowband images and ionization map of NGC 4565. H $\alpha$ (upper left), [O III] (upper right), R-band (lower left), and $[\mathrm{O} \mathrm{III}] / \mathrm{H} \alpha$ (lower right). 
T. P. Robitaille et al.: Gaseous outflows in edge-on Seyfert galaxies, Online Material p 11

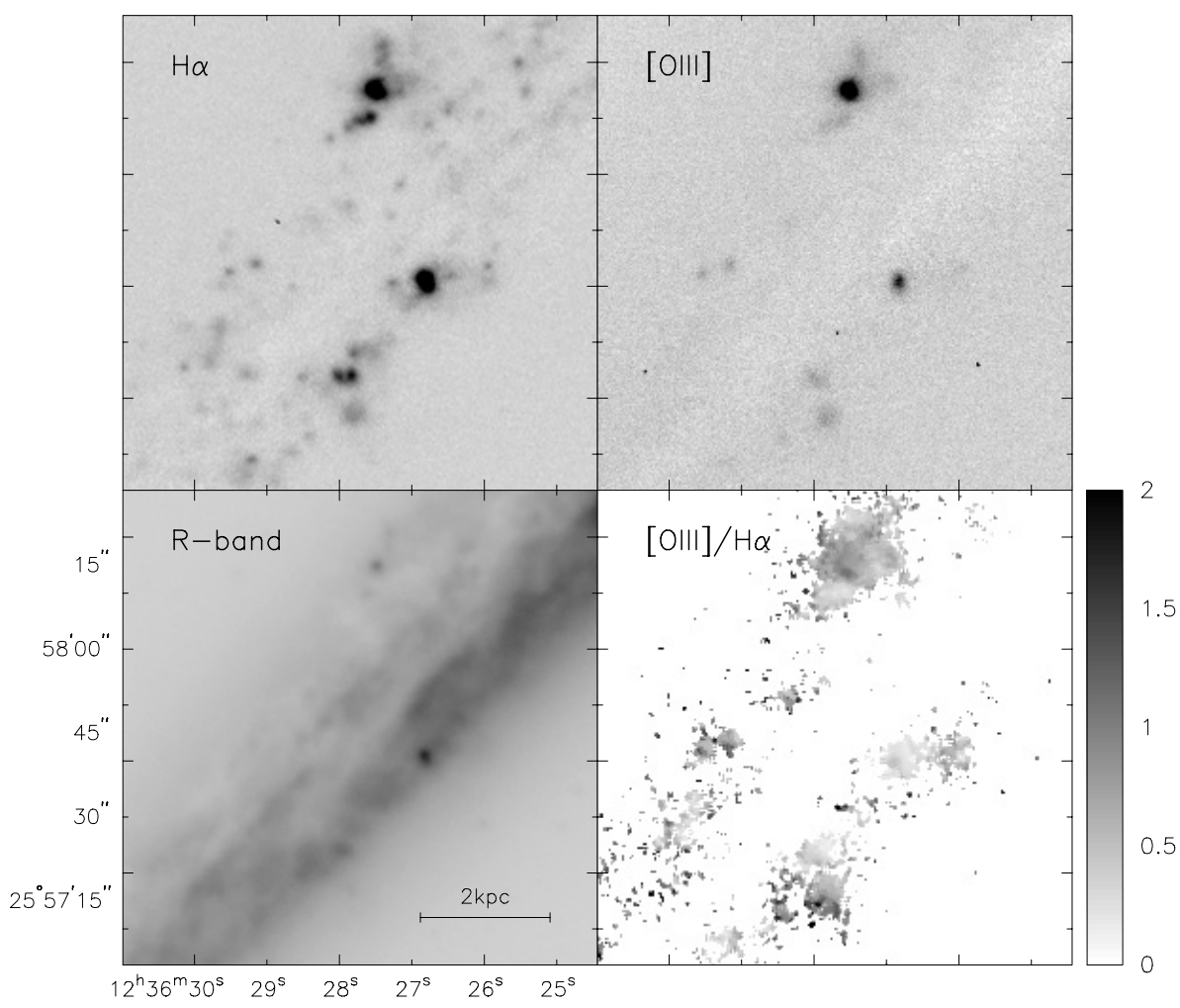

Fig. 21. A zoom-in of Fig. 20 on the southern disk region with prominent $\mathrm{H}$ II regions.

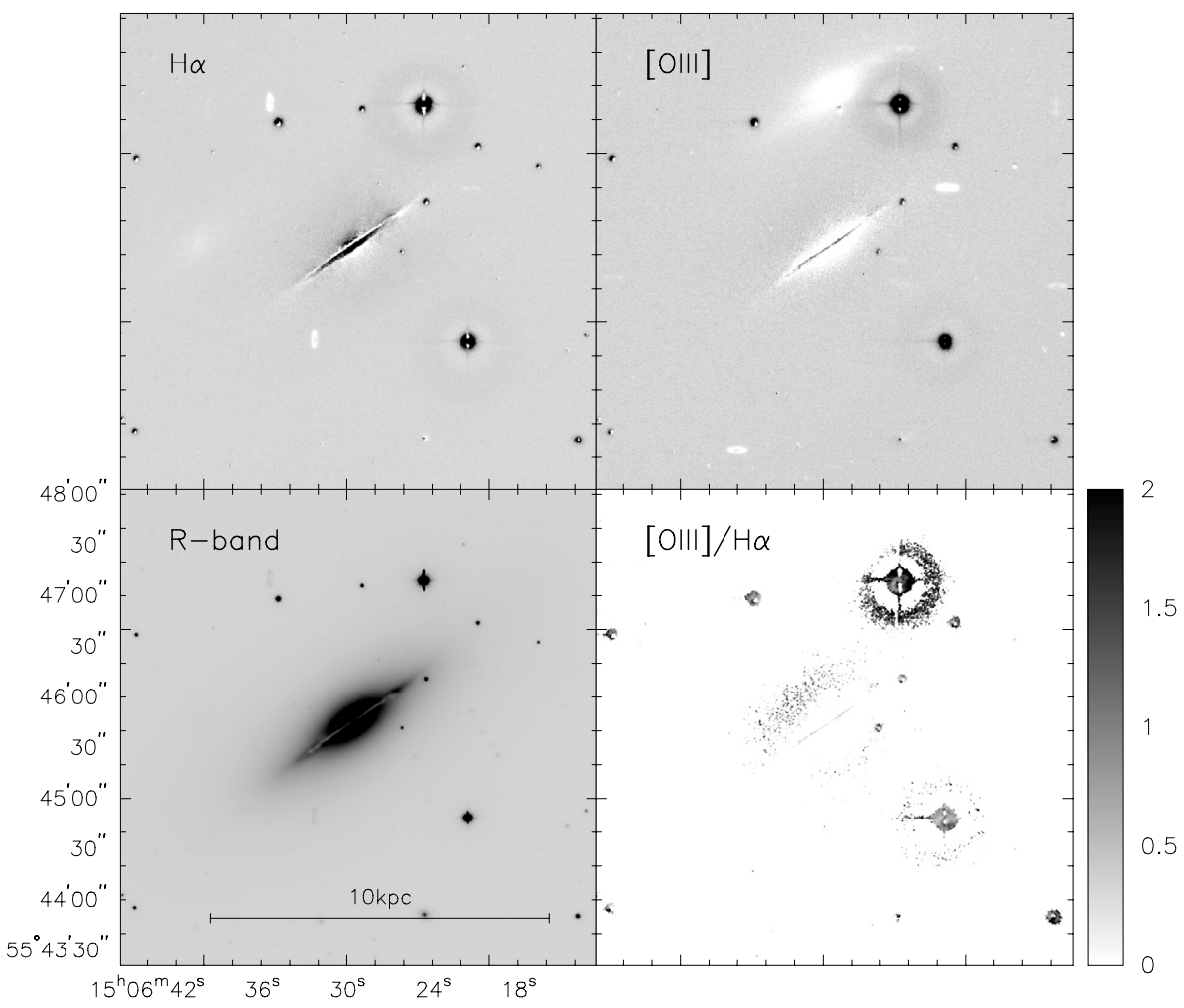

Fig. 22. Broadband, narrowband images and ionization map of NGC 5866. H $\alpha$ (upper left), [O III] (upper right), R-band (lower left), and $[\mathrm{O} \mathrm{III}] / \mathrm{H} \alpha$ (lower right). 
T. P. Robitaille et al.: Gaseous outflows in edge-on Seyfert galaxies, Online Material p 12
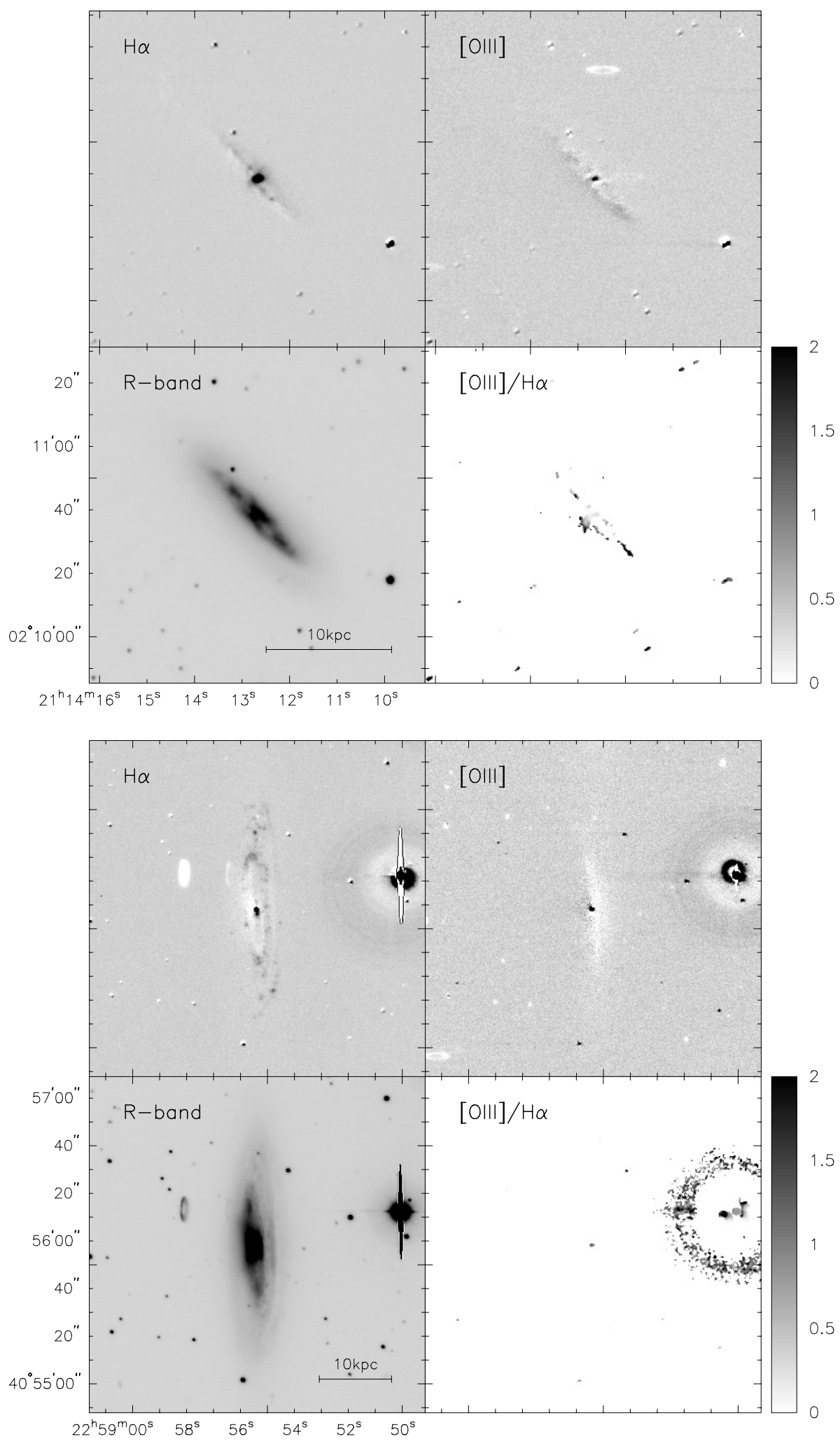

Fig. 23. Broadband, narrowband images and ionization map of IC 1368. H $\alpha$ (upper left), [O III] (upper right), R-band (lower left), and [O III] $/ \mathrm{H} \alpha$ (lower right).
Fig. 24. Broadband, narrowband images and ionization map of UGC 12282. H $\alpha$ (upper left), [O III] (upper right), $R$-band (lower left), and $[\mathrm{OIII}] / \mathrm{H} \alpha$ (lower right). The elongated white/black feature in the $R$-band/H $\alpha$ image to the $\mathrm{E}$ of the disk is a ghost of the bright star close to the right corner of the displayed field of view (see Sect. 2.3.2). 